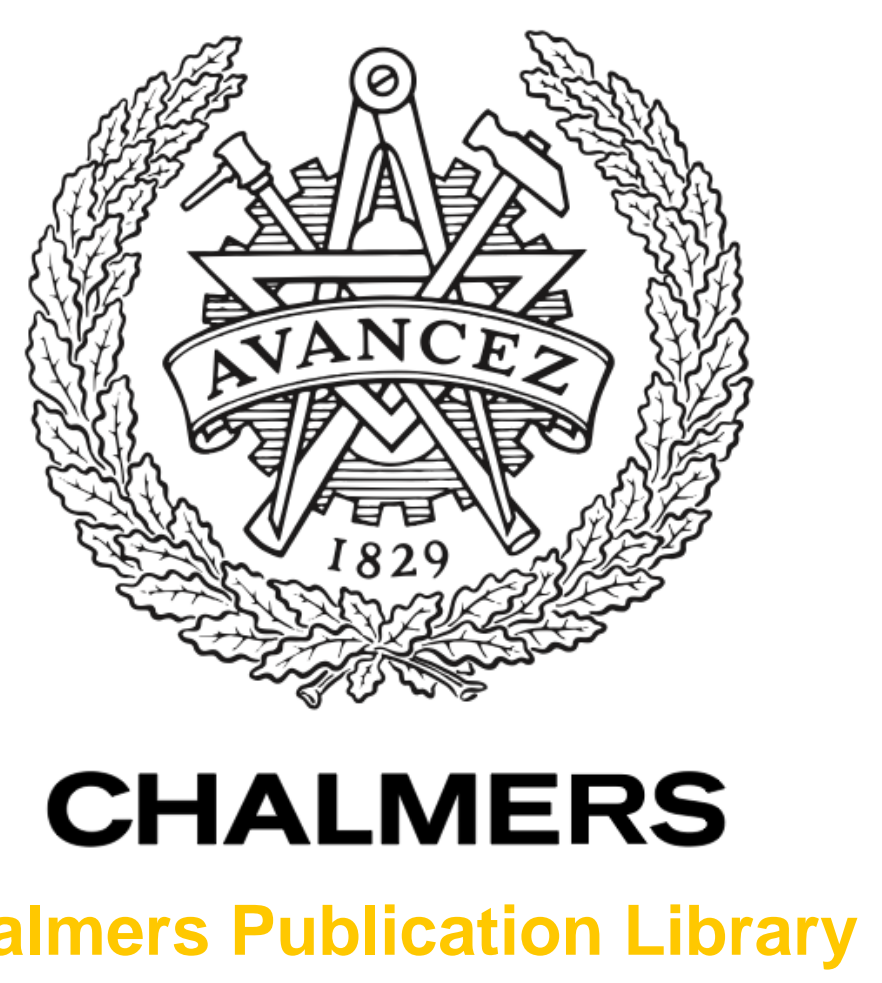

Challmers Publication Library

\title{
Retrieving Layer-Averaged Tropospheric Humidity from Advanced Technology Microwave Sounder Water Vapor Channels
}

This document has been downloaded from Chalmers Publication Library (CPL). It is the author's version of a work that was accepted for publication in:

IEEE Transactions on Geoscience and Remote Sensing (ISSN: 0196-2892)

Citation for the published paper:

Moradi, I. ; Ferraro, R. ; Soden, B. et al. (2015) "Retrieving Layer-Averaged Tropospheric Humidity from Advanced Technology Microwave Sounder Water Vapor Channels". IEEE Transactions on Geoscience and Remote Sensing, vol. 53(12), pp. 6675-6688.

http://dx.doi.org/10.1109/TGRS.2015.2445832

Downloaded from: http://publications.lib.chalmers.se/publication/223275

Notice: Changes introduced as a result of publishing processes such as copy-editing and formatting may not be reflected in this document. For a definitive version of this work, please refer to the published source. Please note that access to the published version might require a subscription. 


\title{
Retrieving Layer Averaged Tropospheric
}

\section{Humidity from Advanced Technology}

\section{Microwave Sounder (ATMS) Water Vapor}

\section{Channels}

\author{
Isaac Moradi, Ralph Ferraro, Brian Soden, Patrick Eriksson, and Phillip Arkin
}

\begin{abstract}
A method is presented to calculate layer-averaged tropospheric humidity (LAH) from the observations of the Advanced Technology Microwave Sounder (ATMS) water vapor channels. The method is based on a linear relation between the satellite brightness temperatures $(\mathrm{Tb})$ and natural logarithm of Jacobian weighted humidity. The empirical coefficients of this linear relation were calculated using different datasets as well as a fast and a line-by-line radiative transfer (RT) model. It was found that the coefficients do not significantly depend on the dataset or the RT model. This $\mathrm{Tb}$ to LAH transformation method can be applied to either original or limb corrected ATMS Tbs. The method was validated using both simulated and observed ATMS Tbs. The systematic difference between the estimated and calculated LAH values was lass than $10 \%$ in most cases. We also tested the transformation method using a fixed Jacobian for each channel. The bias generally increases when fixed Jacobians are used, but there is still a satisfactory agreement between estimated and calculated LAH values. In addition, the spatial distribution of the bias was investigated using the European Center for Medium range Weather Forecasting (ECMWF) Interim Reanalysis (ERA-interim) and collocated ATMS observations. The bias did not indicate any significant regional dependency when actual Jacobians were used, but in the case of fixed Jacobians, the bias generally increased from mid-latitude towards the poles.
\end{abstract}

Index Terms

microwave, remote sensing, water vapor, climate change, hydrology, JPSS

I. Moradi is with Cooperative Institute for Climate and Satellites, Earth System Science Interdisciplinary Center (ESSIC), University of Maryland, and the NOAA Joint Center for Satellite Data Assimilation, College Park, MD 20740, USA. e-mail: imoradi@umd.edu.

R. Ferraro is with the Center for Satellite Applications and Research, National Environmental Satellite, Data, and Information Service, National Oceanic and Atmospheric Administration (NOAA), College Park, MD 20740, USA.

Brian Soden is with Rosenstiel School for Marine and Atmospheric Science, University of Miami, Miami, FL 33149, USA.

Patrick Eriksson is with Department of Earth and Space Sciences, Chalmers University of Technology, Gothenburg, SE-41296, Sweden.

Phillip Arkin is with the Cooperative Institute for Climate and Satellites, Earth System Science Interdisciplinary Center (ESSIC), University of Maryland, College Park, MD 20740, USA. 


\section{INTRODUCTION}

Atmospheric water vapor significantly contributes to the Earth's radiation budget and is a critical variable for climate studies. It is the dominant natural greenhouse gas and the largest known feedback mechanisms for amplifying global warming. Tropospheric humidity both directly and indirectly affects the hydrological cycle [1] as well as strongly contributes to the water vapor feedback, especially in mid-upper troposphere, owing to the low temperatures at those altitudes [2], [3]. All climate models predict that the atmosphere will moisten in response to increasing greenhouse gases. The concentration of water vapor in the upper troposphere is projected to double by the end of the century. However the fractional increase in upper tropospheric water vapor will be roughly three times as large as that for the lower troposphere, even though the relative humidity remains (nearly) constant at all levels. This amplified moistening in response to the surface warming not only highlights the significance of tropospheric water vapor as a feedback mechanism, but also underscores the importance of long-term monitoring of water vapor to the detection and attribution of climate change. The large increase in upper tropospheric water vapor relative to other levels is due to two processes. First, the Clausius-Clapeyron equation dictates that the fractional increase in

saturation vapor pressure $\left(e_{s}\right)$ scales according to $\frac{1}{e_{s}} \frac{d e_{s}}{d T} \propto T^{-2}$. Thus, under constant relative humidity, the change in vapor pressure resulting from a $1 \mathrm{~K}$ warming at $\mathrm{T}=200 \mathrm{~K}$ will be more than twice that obtained at $\mathrm{T}=300 \mathrm{~K}$. Secondly, most climate models predict a reduction in lapse-rate with increasing surface temperature. Thus the upper troposphere tends to warm more than the lower troposphere, further amplifying the upper tropospheric moistening [2], [4], [5].

Satellite and radiosonde data are the main sources that provide information about tropospheric humidity. Radiosonde data have higher vertical resolution than satellite data, but radiosonde data are only available from around 1500 upper air stations while satellite data have global coverage. In addition, previous studies, e.g. [6]-[9], show that radiosonde data are prone to several errors, including contamination, sensor design, calibration, and data processing, particularly in the dry and cold conditions of the middle to upper troposphere. Satellite data have global coverage with fine spatial resolution and are operationally available every few hours. However, satellite observations provide layer averages and do not resolve finer vertical structures of the tropospheric humidity. In addition, satellite data are measured in terms of radiance or brightness temperature $(\mathrm{Tb})$; therefore transformation algorithms are required to convert the satellite Tb's to geophysical variables such as water vapor. The main techniques to retrieve tropospheric humidity from the satellite observations include: (i) inversion methods to retrieve the vertical profiles from layer-averaged radiances, and (ii) direct transformation techniques to directly convert layer-averaged Tb's to layer-averaged humidity. The inversion techniques are computationally expensive, require complicated calculations, a-priori profiles, and some pre-calculated statistical inputs to initialize the retrievals.

A computationally effective technique was introduced by Soden and Bretherton [10], hereafter refereed to as SB93, to transform satellite observations from infrared (IR) sensors into Upper Tropospheric Humidity (UTH). Spencer and Braswell [11] showed that the same technique can be applied to the microwave observations from the Special Sensor Microwave Water Vapor Sounder (SSM-T2) instrument (channels 183 $\pm 1,3,7$ ), but did not provide 
any further analysis such as the empirical coefficients. Buehler and John [12] examined this technique for retrieving UTH from the observations of the Advanced Microwave Sounding Unit (AMSU-B) instrument and provided an in-depth error analysis as well as a table for the empirical coefficients as a function of scan angle for channel $183 \pm 1$ GHz. In this study, we extend the technique to transform the Advanced Technology Microwave Sounder (ATMS) radiances into layer-averaged humidity. The basic relation presented in the current paper is slightly different from the original version introduced by Soden and Bretherton [10], but similar to the relation that was used by Spencer and Braswell [11] and Buehler and John [12]. However, we employ two different techniques to transform satellite observations into tropospheric humidity, including calculating the empirical coefficients of the transformation method as a function of scan angle as well as a technique to correct the satellite data for limb darkening effect then applying the same coefficients to the observations from different beam positions. Buehler and John [12] only examined the relation for $183 \pm 1 \mathrm{GHz}$ which is sensitive to UTH, but we examine the relation for five ATMS water vapor channels which are sensitive to upper to lower tropospheric humidity. We also provide detailed error analysis, examine the effect of the dataset and radiative transfer (RT) models on the empirical coefficients, and provide a comprehensive validation and evaluation including the spatial distribution of the errors. Generally, these conversion techniques are very simple and computationally effective, and unlike inversion techniques do not require extensive radiative transfer calculations. The only input is the calibrated satellite data in terms of brightness temperature.

Section II introduces the satellite and atmospheric datasets that we used in this study, Section III explains the methodology and transformation method, Section IV presents the validation using independent datasets as well as the ATMS observations, and Section V summarizes the study.

\section{SAtellite Instrument AND Atmospheric Profiles}

\section{A. ATMS Instrument}

ATMS is a total-power radiometer and employs 22 channels operating at microwave frequencies from $23.8 \mathrm{GHz}$ to $190.31 \mathrm{GHz}$. ATMS is a cross-track microwave sounder that combines the capabilities of the previous microwave temperature and humidity sounders, i.e. AMSU-A, -B, and Microwave Humidity Sounder (MHS), aboard NOAA's Polar-orbiting Operational Environmental Satellite System (POES). ATMS is planned to fly on the United States next generation polar-orbiting operational environmental satellite system named as the Joint Polar Satellite System (JPSS). This sensor is currently flying on the Suomi National Polar-orbiting Partnership (S-NPP) satellite, launched in October 2011, which is in a Sun-synchronous orbit with the ascending equatorial crossing time at 13:30, an altitude of $824 \mathrm{~km}$, inclination of $97.1^{\circ}$, and an orbital period of 101 minutes. The ATMS water vapor channels operate at $183.31 \pm 7.0 \mathrm{GHz}, 183.31 \pm 4.5 \mathrm{GHz}, 183.31 \pm 3.0 \mathrm{GHz}, 183.31 \pm 1.8 \mathrm{GHz}$, and $183.31 \pm 1.0 \mathrm{GHz}$, hereafter refereed to as $\mathrm{Ch} 7.0, \mathrm{Ch} 4.5, \mathrm{Ch} 3.0, \mathrm{Ch} 1.8$, and $\mathrm{Ch} 1.0$, respectively. These channels are normally numbered as Channels 18-22, respectively. Compared to AMSU-B and MHS, ATMS has two additional water vapor channels (Ch4.5 and Ch1.8). All the ATMS water vapor channels are horizontally polarized. The beamwidth is $1.11^{\circ}$ for all the ATMS water vapor channels. The bandwidth is $2.0 \mathrm{GHz}$ for $\mathrm{Ch} 7.0$ and $\mathrm{Ch} 4.5,1.0 \mathrm{GHz}$ for Ch3.0 and $\mathrm{Ch} 1.8$, and $0.5 \mathrm{GHz}$ for Ch1.0. The instrument has a swath width of approximately $2300 \mathrm{~km}$, with 96 scan positions. 
The antenna is in continuous motion so that the 96 samples are taken on the fly, then each sample is assigned to the mid-point of a sampling interval of about $18 \mathrm{~ms}$. The scan speed is about $61.6^{\circ} \mathrm{s}^{-1}$, so that the angular sampling interval is $1.11^{\circ}$. The instrument scan angle is $0.555^{\circ}$ for the innermost beam positions and $52.725^{\circ}$ for the outermost beam positions [13], [14].

\section{B. Atmospheric Datasets}

Four different global datasets were used to conduct the radiative transfer simulations, that are presented in this section. We also combined all the datasets together in order to calculate the empirical coefficients of the transformation algorithm and limb-correction method. We refer to this dataset as the combined dataset throughout the rest of the paper.

The SeeBor dataset consists of approximately 12,000 global profiles of temperature, moisture, and ozone at 101 pressure levels for clear sky conditions sampled from NOAA-88 (44\% of total profiles), ECMWF (34\%), the Thermodynamic Initial Guess Retrieval (TIGR) (9\%), ozonesondes from 8 NOAA Climate Monitoring and Diagnostics Laboratory (8\%), and radiosonde data from the Sahara desert (5\%) [15]. This dataset is sampled to cover a wide range of temperature and humidity variations.

The European Organization for the Exploitation of Meteorological Satellites (EUMETSAT) Satellite Application Facility on Numerical Weather Prediction (NWP-SAF) datasets include atmospheric temperature, water vapor, and ozone simulated by the European Center for Medium-Range Weather Forecasts (ECMWF) system. Each SAF database contains 5000 profiles available at 91 pressure levels. In this study, we used two different SAF datasets known as SAF-Q and SAF-T which are sampled to cover large humidity (SAF-Q) and temperature (SAF-T) variations [16].

The Atmospheric Radiation Measurement (ARM) Climate Research Facility is a global change research program supported by the United States Department of Energy since 1989. We used radiosonde data from the following ARM stations: the Tropical Western Pacific stations (TWP-C1, -C2, and -C3) located in Manus Island (Papua New Guinea), Nauru Island (the Republic of Nauru), and Darwin (Australia), respectively, Southern Great Plains (SGP-C1) located in Lamont (Oklahoma, USA), and North Slope of Alaska (NSA-C1) located in Barrow, USA [17].

\section{Methodology and Transformation Method}

The ATMS water vapor sounding channels are sensitive to the amount of water vapor in different layers of the atmosphere; therefore these channels can be used to retrieve the layer-averaged tropospheric humidity in different layers of the troposphere. The sensitivity of the microwave water vapor channels to the concentration of water vapor at different altitudes of the atmosphere can be defined using Jacobians. Jacobians are mathematically the partial derivative of radiance with respect to the atmospheric parameters influencing the observed radiance. Jacobians can be simply calculated using a perturbation method which is a good approximation for small variations in the amount of water vapor. In the perturbation method, the water vapor Jacobians $(K)$ are defined as follows: 


$$
K_{j}=\frac{T b\left(q_{j}+\Delta q_{j}\right)-T b\left(q_{j}-\Delta q_{j}\right)}{2 \Delta q_{j}}
$$

where, $\mathrm{q}$ is water vapor at the $\mathrm{j}$-th level, and $\Delta q$ is a small number (here set to $0.05 q$ ). This relation describes change in Tb (in Kelvin) due to a small change in the water vapor concentration [18]. The unit of Jacobians is defined based on the humidity quantity that it perturbed. In this study, volume mixing ratio is used but also relative humidity or mass mixing ratio may be perturbed in Equation 1. Negative Jacobians mean that any increase in the water vapor concentration lowers the observed radiance $(\mathrm{Tb})$ and vice verse. It should be noted that due to the limitation in numerical calculations, the Jacobians calculated using the perturbation method are approximative.

Figure 1 shows the Jacobians of the ATMS water vapor channels for a subarctic winter, mid-latitude winter, subarctic summer, and a tropical profile. As indicated in Figure 1, the peak altitude of the Jacobians is a function of precipitable water vapor (PWV), beam position, and the frequency of the channel. The peak is at a higher altitude for the channels which operate at the frequencies closer to the center of the water vapor absorption line at $183 \mathrm{GHz}$. Jacobians may become positive near the surface especially in dry conditions for the lower channels. In this case, the observed radiance is affected by the emitted radiance from the surface which is a function of the surface emissivity. For instance, Figure 1 shows that for a subarctic winter profile which is relatively dry, PWV $=5 \mathrm{~kg} \mathrm{~m}^{-2}$, the three lower water vapor channels, i.e. Ch3.0, Ch4.5 and Ch7.0, are affected by the surface emissivity, but for a tropical profile which is relatively humid (PWV $=41 \mathrm{~kg} \mathrm{~m}^{-2}$ ), none of the ATMS water vapor channels are affected by the surface emissivity. Accurate surface emissivity data are required to simulate satellite $\mathrm{Tb}$ 's when the radiances are affected by the surface; besides when the Jacobians peak near the surface, the satellite observations become more sensitive to PWV rather than relative humidity. Since current microwave surface emissivity data do not have high accuracy, see e.g., [19], and also the aim of this study is not to retrieve PWV from the ATMS observations, we limited our study to the profiles where satellite Tb's are not affected by the surface emissivity.

Figure 2 shows the surface contribution to the simulated ATMS Tb's as a function of PWV. Surface contribution was defined as the difference between simulated Tb's when emissivity varies from 0 to 1 [7]. As shown in Figure 2, the ATMS Tb's are only affected by the surface emissivity if PWV is less than $30,20,10,7$, and $5 \mathrm{~kg} \mathrm{~m}^{-2}$ for Ch7.0, Ch4.5, Ch3.0, Ch1.8, and Ch1.0, respectively. We used these thresholds to screen out all the ATMS observations or simulations that are affected by the surface emissivity. This filter removes $60 \%, 48 \%, 32 \%, 21 \%$, and $18 \%$ of the combined dataset profiles for $\mathrm{Ch} 7.0, \mathrm{Ch} 4.5, \mathrm{Ch} 3.0, \mathrm{Ch} 1.8$, and $\mathrm{Ch} 1.0$, respectively. ATMS observations from the channels operating at $23.8 \mathrm{GHz}$ and $31.4 \mathrm{GHz}$ can be used to derive PWV, when the PWV measurements are not available [20].

Layer averaged tropospheric humidity (LAH) is calculated using convolution of the Jacobians and the humidity profiles:

$$
L A H=\frac{\sum_{j} K_{j} R H_{j}}{\sum_{j} K_{j}}
$$

where, RH shows the relative humidity with respect to water at the level $\mathrm{j}$. In this study, in addition to using the 
actual Jacobians to calculate the LAH, we also used a fixed Jacobian for each channel, Jacobians shown in Figure 1 for a profile with PWV $=28 \mathrm{~kg} \cdot \mathrm{m}^{-2}$, to calculate the $\mathrm{LAH}$, because it is easier to compare the LAH values averaged over a fixed layer with the in-situ data or climate models field.

A primary relation was introduced by Soden and Bretherton [10] to derive UTH from the infrared observations of Geostationary Operational Environmental Satellite (GOES) as follows:

$$
\ln \left(\frac{U T H}{\cos \theta}\right)=a+b \times T b
$$

where $\ln$ is the natural logarithm, $\theta$ is the earth incidence angle (EIA), $a$ and $b$ are the empirical coefficients to be determined, and $\mathrm{Tb}$ is the observed brightness temperature in Kelvin. In the current study, we employ a modified version of Equation 3 which was proposed by Spencer and Braswell [11] for SSM-T2 observations and examined by Buehler and John [12] for deriving UTH from AMSU-B brightness temperatures:

$$
\ln (L A H)=a+b \times T b
$$

First, Jacobians and Tb's are calculated using a radiative transfer model and atmospheric profiles stated in Section II-B, then LAH values are calculated by the convolution of Jacobians and relative humidity profiles, see Equation 2. Finally, the empirical coefficients are calculated by relating $\ln (L A H)$ to $\mathrm{Tb}$ using Equation 4. Practically, once the coefficients are calculated, they can be used in conjunction with the observed satellite Tb's to calculate LAH. As mentioned before, it is essential to only use the data that are not affected by the surface emissivity.

ATMS Tb's systematically decrease from nadir to the edge of the scan which is called limb-darkening effect. Limb darkening is caused by increase in the optical path length related to the Earth Incidence Angle (EIA), see Appendix A for the definition of EIA. ATMS Jacobians also move to higher altitudes from nadir to the edges of the scan due to the same effect, see Figure 1. The overall impact of these scan angle dependencies is that the empirical coefficients in Equation 4 depend on the beam position. Thus, it is required to either calculate the coefficients for each beam position or correct the ATMS Tb's for the limb effect [21]. In the latter case, the same coefficients calculated using the nadir Tb's can be applied to the limb adjusted Tb's. It should be noted that in the original relation introduced by Soden and Bretherton [10], $\cos \theta$ accounts for the scan angle dependency of UTH.

Buehler and John [12] calculated the empirical coefficients ( $a$ and $b$ ) for individual beam positions and directly used the tabulated values to estimate UTH from AMSU-B observations. However, this method is not practical since it introduces 480 coefficients for the ATMS water vapor channels. Instead, we relate the coefficients for different beam positions to EIA as follows:

$$
\begin{array}{r}
a(\theta)=a_{1}+a_{2} \times \ln (\cos \theta) \\
b(\theta)=b_{1}+b_{2} \times \ln (\cos \theta)
\end{array}
$$

where, $a_{1}, a_{2}, b_{1}$ and $b_{2}$ are empirical coefficients calculated for each channel and $\theta$ is EIA. 
We also developed a limb-correction method similar to Chung et al. [21]. As shown in Figure 3, the limb darkening $(\Delta T b)$ which is calculated as the difference between $\mathrm{Tb}$ given at any $\theta$ and nadir $\mathrm{Tb}$, is a function of EIA and it is stronger for the channels operating at the frequencies closer to the water vapor absorption line at $183 \mathrm{GHz}$. We rewrite the limb-correction relation suggested by Chung et al. [21] as follows:

$$
\begin{gathered}
T b^{n}=T b(\theta)-\Delta T b \\
\Delta T b=c \times \ln (\cos \theta)
\end{gathered}
$$

where, $T b^{n}$ is $\mathrm{Tb}$ at sub-nadir footprint, and $T b(\theta)$ is $\mathrm{Tb}$ at any given $\theta$. The values for the coefficient $\mathrm{c}$ are presented in Table I for different ATMS water vapor channels. As shown, the coefficients do not significantly depend on dataset or the RT model. It should be noted that these values can only be applied to the ATMS Tb's that are not affected by the surface emissivity. We hereafter refer to the first method, which is based on calculating empirical coefficients as a function of $\theta$, as coefficient adjustment (CA) method and to the second method, which is based on adjusting ATMS Tb's for limb-effect, as Tb limb adjustment (TLA) method.

We used Atmospheric Radiative Transfer Simulator (ARTS) as the line-by-line (LBL) model [22] and the Community Radiative Transfer Model (CRTM) [23] as the fast radiative transfer model. ARTS is a general forward model, but its development has a special emphasis on atmospheric sounding using microwave instruments. For water vapor absorption the model of Rosenkranz [24] was selected. CRTM has been widely used across NOAA in recent years for operational purposes such as data assimilation and numerical weather prediction. Fast RT models are computationally faster than the LBL models but normally less accurate, because fast RT models estimate the absorption coefficients based on a look-up table using a range of predictors and do not directly calculate them using the spectroscopy databases and RT theory [25]. However, the fast RT models have greatly improved over the past decade [26]. In this study, we used a K-Matrix technique for the fast model and an analytical derivation for the LBL model to calculate the Jacobians.

\section{RESUlTS AND DisCUSSION}

In this section, we first present the calculated coefficients for the Tb to LAH transformation method and discuss the impact of the RT model as well as the atmospheric profiles on the calculated coefficients, then discuss the validation of the transformation method using independent datasets. We use the terms "estimated LAH" for the LAH values estimated using the transformation method (Equation 4) and "calculated LAH" for the LAH values calculated using the convolution of Jacobians and relative humidity profiles (Equation 2).

\section{A. Empirical Coefficients}

Figure 4 shows simulated satellite Tb's for the ATMS water vapor channels versus the LAH values calculated using Jacobians. Figure 4 only shows Tb and LAH values for the nadir beam positions. We used the PWV thresholds, see Section III, to screen out the Tb's that were affected by the surface emissivity. The results presented in Figure 
4 show a strong relation between $\mathrm{Tb}$ and LAH for all the ATMS water vapor channels. The calculated coefficients for Equation 4 are slightly different for different water vapor channels. Since there is an anti-correlation between tropospheric humidity and satellite Tb's, the slope of the fitted line (coefficient $b$ in Equation 4) is always negative. Figure 4 also compares the effect of different RT models (LBL vs. fast model) as well as different atmospheric datasets (combined dataset vs. SeeBor) on the relation between $\mathrm{Tb}$ and LAH. It was found that the coefficients only slightly depend on the datasets or the RT model. Figure 5 indicates the difference between LAH values estimated using different set of empirical coefficients. The values shown in this figure are the difference between the LAH values calculated using the combined dataset (LBL model) and the LAH values calculated using other datasets or RT models. As shown, the differences are less than $0.1 \mathrm{RH}$ which is about $10 \%$ in relative unit.

The values calculated using combined dataset and the LBL model for Ch7.0, Ch4.5, Ch3.0, Ch1.8, and Ch1.0 are 16.9, 16.1, 15.9, 16.4, and 16.5 for coefficient $a$ and $-0.064,-0.063,-0.064,-0.068$, and -0.070 for coefficient $b$, respectively. We used two different models, a LBL and a fast RT model, to calculate the empirical coefficients. As shown in Figures 4 and 5, the magnitude of the differences between the models is as large as that introduced by different datasets. Overall, there is slightly a better agreement (higher correlation) between $\mathrm{Tb}$ and $\ln (L A H)$ simulated using combined dataset and the LBL model. Therefore, we adopted the coefficients calculated using this combination for validating the transformation method. Coefficients calculated using the combined dataset and the LBL model for Ch1.0 are consistent with Buehler and John [12] who reported 16.47 for the coefficient $a$ and -0.070 for the coefficient $b$ for a similar channel on AMSU-B instrument. As explained before, the empirical coefficients depend on the beam position, see Figure 6, so that a logarithmic relation was suggested to calculate the empirical coefficients for different beam positions as a function of EIA. The coefficients for these logarithmic relations are given in Table II for both actual and fixed Jacobians. These values can be inserted into Equation 5 to calculate the empirical coefficients of the transformation method for different beam positions. Table II also shows the coefficients $a$ and $b$ for the nadir beam positions. These coefficients can be applied to the limb-adjusted ATMS Tb's, independent of the beam position.

\section{B. Validation}

We used the ARM radiosonde profiles as well as globally sampled profiles, such as SAF and SeeBor, see Section II-B, to validate the results. As a first test, the LAH values were estimated from ATMS Tb's simulated using the LBL model. Secondly, the actual ATMS observations collocated with the ARM radiosonde profiles were used to estimate LAH. In either case, the estimated LAH values were compared with the Jacobian weighted LAH values.

\section{Simulated ATMS Brightness Temperatures}

The vertical profiles of temperature and humidity were used to simulate both ATMS Tb's as well as the corresponding Jacobians. Figure 7 shows the LAH values estimated from the ATMS simulated Tb's versus the Jacobian weighted LAH values, both ATMS Tb's and Jacobians were simulated using ARM profiles. We used both actual Jacobians as well as the fixed Jacobians mentioned in Section III, to calculate LAH from the humidity 
profiles. As shown in Figure 7, in the case of actual Jacobians, estimated and calculated values are generally in very good agreement, but the method slightly underestimate tropospheric humidity in moist conditions. The scatterplots show a larger standard deviation in moist conditions than in dry conditions.

The statistics for this comparison are shown in Table III for different datasets. Difference between estimated and calculated LAH values, when the LBL model and actual Jacobians are used, is less than $10 \%$ in all cases. The statistics are very similar for the LAH values estimated using either the CA method or the TLA method. This indicates that either of the CA or TLA methods can be equally used to estimate LAH. The bias between estimated LAH (using either CA or TLA methods) and calculated LAH values is less than $-7 \%$ for all the channels at the ARM stations. In the case of LAH values calculated using fixed Jacobians, the bias is generally larger than that for actual Jacobians, but for the ARM data the bias is still less than $-5 \%$ for all the channels. The bias between the estimated and calculated LAH values using the SeeBor and SAF-Q datasets ranges, respectively, from $4 \%$ to $9 \%$ and $-3 \%$ to $-8 \%$ for the actual Jacobians and from $8 \%$ to $10 \%$ and $-4 \%$ to $-9 \%$ for the fixed Jacobians.

Table III also shows the standard deviation of the differences between estimated and calculated LAH values. The standard deviations for the actual Jacobians and the LBL model range from 0.08 to $0.09 \mathrm{RH}$ for the ARM dataset, from 0.05 to $0.07 \mathrm{RH}$ for the SAF-Q dataset, from 0.06 to $0.07 \mathrm{RH}$ for the SAF-T dataset, and from 0.05 to $0.07 \mathrm{RH}$ for the SeeBor dataset. The standard deviations for the fixed Jacobians are greater than those for actual Jacobians and range from 0.08 to $0.13 \mathrm{RH}$ for the ARM dataset, from from 0.10 to $0.13 \mathrm{RH}$ for the SAF-Q dataset, from 0.08 to $0.11 \mathrm{RH}$ for the SAF-T dataset, and from 0.07 to $0.10 \mathrm{RH}$ for the SeeBor dataset. Thus, the agreement between the estimated and calculated LAH values using fixed Jacobians is generally less than that for actual Jacobians. In some cases, e.g., the ARM dataset, the bias for the fixed Jacobians is less than that for the actual Jacobians, however, the standard deviations are generally higher for the fixed Jacobians than for the actual Jacobians. Table III also shows the simulations conducted using a fast model. The fast model generally shows larger biases than the LBL model. In this case, the bias between the calculated and estimated LAH values ranges from $-4 \%$ to $1 \%$ for the ARM dataset, from $-3 \%$ to $12 \%$ for the SAF-Q dataset, from $-2 \%$ to $8 \%$ for the SAF-T dataset, and from $7 \%$ to $18 \%$ for the SeeBor dataset. The CA and TLA methods also indicate similar results when the fast model is used to conduct the RT simulations. The main reason for the better agreement between the estimated and calculated LAH values using the LBL simulations should be that the empirical coefficients employed to estimate the LAH from simulated Tb's were calculated using the same model. Since the bias depends on LAH, for instance see Figure 7, the slope of the fitted line is also included in Table III. In most cases, the slope ranges between 0.9 to 1.1 and does not depend on the RT model or other factors such as limb-correction or Jacobians. However, the slop slightly depends on the channel. Generally, the slop is closer to unity for lower tropospheric channels than for upper tropospheric channels.

\section{Observed ATMS Brightness Temperatures}

The ATMS observations were collocated with the ARM radiosonde profiles for the time period of January 2012 through August 2013. The collocation criteria was less than $100 \mathrm{~km}$ distance between the ATMS footprints and 
the radiosonde station and less than 3 hours time difference between the ATMS scanning time and the radiosonde launch time. Since radiosondes drift while ascending through the troposphere, we compared each radiosonde launch with the average LAH $(\mathrm{Tb})$ of a target area with a radius of $100 \mathrm{~km}$. It should be noted that we first estimated LAH from individual Tb's within the target area then averaged the LAH values. In addition, the difference between Tb's from different channels was used to filter out the observations affected by the clouds [27], [28]. We used the difference between $\mathrm{Tb}$ of $\mathrm{Ch} 7.0\left(\mathrm{~Tb}_{7.0}\right)$ and $\mathrm{Tb}$ of $\mathrm{Ch} 4.5\left(\mathrm{~Tb}_{4.5}\right), \Delta T b=T b_{7.0}-T b_{4.5}$ to identify and exclude the ATMS observations affected by the clouds. In clear-sky conditions, $\mathrm{Tb}_{7.0}$ is higher than $\mathrm{Tb}_{4.5}$ but in the presence of optically thick clouds, $\mathrm{Tb}_{7.0}$ is more affected by clouds than $\mathrm{Tb}_{4.5}$, so that $\mathrm{Tb}_{7.0}$ becomes closer or lower than $\mathrm{Tb}_{4.5}$. We excluded all the collocations with at least one $\Delta T b$ within the target area less than $3 \mathrm{~K}$. After collocating radiosonde and satellite observations, the empirical relation (Equation 4) was used to estimate LAH from the ATMS observations and the LBL Jacobians were used to calculate LAH from the ARM radiosonde profiles.

Table IV presents the mean (bias) and standard deviation of differences between the estimated and calculated LAH values. The bias is less than $10 \%$ in most cases when the actual Jacobians are used to calculate LAH. LAH values calculated using either limb-corrected Tb's or original $\mathrm{Tb}$ 's indicate the same accuracy compared to the calculated LAH values. The NSA-C1 station, located in Alaska, shows the largest biases which is related to the dry climate of NSA-C1. Although we applied the PWV thresholds to filter out the surface affected radiances; yet in the NSA-C1 atmospheric conditions, some of the collocated datapoints may still be affected by the surface emissivity. In addition, at the NSA-C1 station, most of the collocated datapoints are screened out by the PWV filter, see Table IV, which affects the reliability of the statistics. The relative bias is generally larger for the TLA method than for the CA method which can also be explained by the effect of surface emissivity on the limb correction. The biases between the estimated LAH values and the LAH values calculated using the fixed Jacobians are also shown in Table IV. In this case, the biases are larger than those for actual Jacobians. The bias is between $20 \%$ to $35 \%$ at NSA-C1 and SGP-C1, and less than $20 \%$ at the TWP stations. Standard deviation of the differences between estimated and calculated LAH values are also shown in Table IV. The standard deviation of the LAH values calculated using fixed Jacobians is larger than those calculated using actual Jacobians which is expected as it was explained before. The slope of the fitted lines is also reported in Table IV. Slope ranges between 0.7 to 0.8 which shows an overestimate for low LAH values and an underestimate for high LAH values. The slope is generally closer to unity for lower level channels than for upper level channels. But the slope does not significantly depend on the RT model or other factors such as Jacobian and limb correction.

Table IV also shows the differences between the ATMS Tbs and the RT simulated Tb's. Obviously about 1 $\mathrm{K}$ mean difference is observed between the simulated and observed ATMS Tb's. It should be noted that the Tb difference affects the LAH comparison as well. This difference is caused by several factors including systematic errors in the RT calculations, sampling error, a known dry bias in the radiosonde data, the effect of optically thin clouds which are not screened out by the cloud filter, as well as inaccuracy in the ATMS calibration. Therefore, it is estimated that a large portion of the systematic difference between the estimated and calculated LAH values is related to the errors aforementioned. These errors can be simply neglected in the LAH comparison by adjusting 
the ATMS Tb's for the difference between simulated Tb's and observed Tb's. Table V shows the statistics for the LAH comparison based on adjusted ATMS Tb's. Obviously, the error has systematically decreased at most stations, excluding TWP-C1 where the error has slightly increased.

\section{Spatial Distribution of Bias}

We used two months of data, August 2012 and January 2013, from the European Center for Medium range Weather Forecasting (ECMWF) Interim Reanalysis (ERA-interim) with collocated ATMS observations to evaluate the spatial distribution and regional dependency of the bias. We used a temporal threshold of $1 \mathrm{~h}$ and spatial threshold of $100 \mathrm{~km}$ for collocating ATMS and ERA-interim data. We excluded all the ATMS observations that were either affected by the clouds or the surface emissivity. The LAH values were calculated using the ERA-interim humidity profiles and corresponding Jacobians and the collocated ATMS observations were used to estimate the LAH. Figure 8 shows the difference between the estimated and calculated LAH values. The ERA-interim data are available only every 6 hours in UTC time, but the ATMS ascending and descending overpass times are at 13:30 and 1:30 local time. Therefore, most collocations occur near the meridians where the local time is around 01:30 or 13:30 for ERA-interim data. These meridians are $22.5^{\circ} \mathrm{E}$ (ERA-interim 00:00 UTC collocated with ATMS descending orbit), $112.5^{\circ} \mathrm{E}$ (ERA-interim 06:00 UTC collocated with ATMS ascending orbit), 157.5 ${ }^{\circ} \mathrm{W}$ (ERA-interim 12:00 UTC collocated with ATMS descending orbit), 67.5 $\mathrm{W}$ (ERA-interim 18:00 UTC collocated with ATMS ascending orbit). Most collocations for the lower tropospheric channels, especially outside tropical region, are removed by the PWV filter, thus these channels can only be validated in very humid regions. The bias (ATMS minus ERA-interim) is generally positive and less than $20 \%$ for all the channels. The bias is less than $10 \%$ for Ch1.0 and Ch1.8. ATMS channels sensitive to lower troposphere show a larger bias than those channels sensitive to mid and upper troposphere. Besides, the bias generally increases from mid-latitudes toward the poles for the lower channels. This feature can be due to surface effect as the PWV filter may not completely remove the profiles affected by the surface emissivity. Figure 8 also shows the results when the LAH values were calculated using fixed Jacobians. In the tropical region, the results for the fixed Jacobians are similar to those obtained using actual Jacobians, but outside tropical region the bias increases towards the poles for the fixed Jacobians. So that the bias for the fixed Jacobians is less than $10 \%$ in tropical and mid-latitude regions, except for Ch7.0 with a bias less than $20 \%$ in tropical region, but increases to up to $30 \%$ in the polar region. This is due to the fact that the fixed Jacobians that we used to derive the empirical coefficients were simulated using a profile with $\mathrm{PWV}=28 \mathrm{~kg} \mathrm{~m}^{-2}$. Therefore, in the polar regions, the ATMS observations point to a layer lower than that for fixed Jacobians which causes an additional bias in the estimated LAH values. Overall, the positive bias (ATMS LAH minus ERA LAH) is mainly related to the systematic difference between ERA-interim and ATMS data. For instance, Figure 9 shows the difference between ATMS observations and Tb's simulated using ERA-interim temperature and humidity profiles for Ch7.0 and Ch1.0. As shown, the ATMS Tb's are systematically lower than ERA-interim simulated Tb's which translates to a systematic wet bias in humidity space. 


\section{Error Analysis}

Based on the comparison between LAH values estimated from simulated Tb's and the LAH values calculated using corresponding humidity profiles and Jacobians, the overall methodological error is estimated to be less than $10 \%$ when actual Jacobians are utilized. However, the error is much larger, up to $30 \%$, when fixed Jacobians are used. In addition, the error in satellite observations should be estimated and be added to the error estimate. Based on RT calculations, it is estimated that $1 \mathrm{~K}$ error in satellite Tb's translates to about $10 \%$ error in terms of relative humidity. This ratio was calculated by perturbing ARM radiosonde humidity profiles by $5 \%$ and $10 \%$ and then computing the impact on ATMS simulated Tb's which was $0.5 \mathrm{~K}$ and $1 \mathrm{~K}$, respectively. The error in satellite observations is estimated to be less than $0.5 \mathrm{~K}$ if the observations are carefully calibrated and bias corrected. This error translates to about $5 \%$ in terms of relative humidity. However, it should be noted that the error in satellite data can be very large, especially due to sensor degradation and drift in the sensor calibration, if the data are not well calibrated.

Several sources contribute to the systematic and random errors in the calculated and estimated LAH values that are explained here. The systematic errors are caused mainly by RT and instrumental errors. The RT error due to spectroscopy and other RT model problems is estimated to be about $0.3 \mathrm{~K}$ based on the difference between the LBL and the fast RT models. Error in the RT simulations for the ATMS instrument, for instance due to imperfect antenna pattern as well as assuming rectangular passbands in the RT calculations while the actual passbands might not be rectangular, and inaccuracy in the ATMS observations due to several sources such as error in the calibration coefficients, are estimated to be less than $0.5 \mathrm{~K}$ based on initial evaluation of ATMS observations. The systematic error in the transformation method is estimated to be about $0.1 \mathrm{RH}$. This error was estimated based on the difference between the LAH values estimated using empirical coefficients determined from different datasets. Another source of error especially in comparing actual ATMS Tb and collocated radiosonde data is from sampling error, because the satellite overpass time and radiosonde launch time are normally fixed with respect to each other, therefore, the time difference normally causes a systematic bias that depends on the overpass times as well as diurnal variation of LAH. Error in radiosonde data is also estimated to be around $5 \%$ which is mainly due to a known dry bias in Vaisala sensors that are used at the ARM stations. The error is larger for the daytime data because of the daytime radiation dry bias but we only used night-time data. It should be noted that some of these systematic errors may cancel each other due to opposite sign of the error.

In addition to the systematic errors, there are also several sources of random errors including random error in RT calculations, noise in the ATMS observations, and random errors in radiosonde measurements. The random errors are normally canceled out after averaging when comparing estimated and calculated LAH values. It should be noted that most of the systematic errors are also canceled out when we use simulated Tb's, instead of measured $\mathrm{Tb}$ 's, to validate the transformation method. In the case of simulated Tb's, the RT error is negligible since we use the same model to calculate both Tb's and Jacobians, and is canceled out after averaging, the ATMS observations are not involved in the comparison, there is no sampling error, and error in the radiosonde data does not matter 
since the same profiles are used for calculating LAH and also simulating the ATMS Tb's. Thus, we believe that the validation that was conducted using the simulated ATMS Tb's better represent the intrinsic accuracy of the transformation method.

\section{CONCluding Remarks}

Atmospheric humidity is the main greenhouse gas and has a significant effect on the Earth radiation budget and atmospheric rivers. One of the main sources to derive information about atmospheric humidity is the satellite observations from the microwave instruments. The Advanced Technology Microwave Sounder (ATMS) is a crosstrack scanning radiometer with 22 channels, of which 5 channels function at the frequencies close to the water vapor absorption line at $183 \mathrm{GHz}$. These channels operate at $183.31 \pm 7.0 \mathrm{GHz}, 183.31 \pm 4.5 \mathrm{GHz}, 183.31 \pm 3.0 \mathrm{GHz}$, $183.31 \pm 1.8 \mathrm{GHz}$, and $183.31 \pm 1.0 \mathrm{GHz}$ (numbered as $18-22$, respectively) and are sensitive to the tropospheric humidity in lower to upper troposphere.

A simple linear relation is presented to directly transform the ATMS observations into layer-averaged humidity (LAH). Unlike inversion methods, radiative transfer calculations are not required to utilize the method and the ATMS observations can be directly transformed into layer-averaged tropospheric humidity. The LAH values calculated using this method can be used, for instance, to validate climate models, study atmospheric rivers, evaluate the effect of tropospheric humidity on outgoing longwave radiation, or to investigate the global atmospheric water cycle. The empirical coefficients of this relation are presented for all the ATMS water vapor channel.

The method was originally introduced by Soden and Bretherton [10] for calculating the upper tropospheric humidity from the infrared satellite observations. A modified version of this method was later examined by Spencer and Braswell [11] and Buehler and John [12] for microwave observations. However, in the current study we employed different techniques to transform ATMS observations into LAH, including a technique to calculate the coefficients for different beam positions as a function of earth incidence angle as well as a technique to correct the satellite data for limb darkening then applying the same coefficients to the observations from different beam positions. Buehler and John [12] only examined the relation for $183 \pm 1 \mathrm{GHz}$ which is sensitive to UTH, but we examine the relation for five ATMS water vapor channels which are sensitive to upper to lower tropospheric humidity. We also examine the effect of the datasets and radiative transfer models on the empirical coefficients. In addition, we provide an extensive error analysis and a comprehensive validation and evaluation including the spatial distribution of the errors.

Since the lower tropospheric channels can be very sensitive to the surface emissivity especially in dry conditions; a filter based on total precipitable water vapor (PWV) was introduced to filter-out all the observations that are affected by the surface emissivity. Because of the limb darkening effect, the empirical coefficients of the transformation method depend on the beam position. Hence, a logarithmic relation between the lapse in ATMS Tb's due to limbeffect and earth incidence angle (EIA) is presented to adjust the ATMS Tb's for limb-darkening, then the same coefficients calculated for the nadir beam positions can be applied to the entire dataset. In addition, a relation is presented to calculate the empirical coefficients as a function of EIA. 
The results were validated using both simulated ATMS Tb's as well as actual ATMS observations. The systematic difference between estimated LAH from the ATMS Tb's and calculated values using Jacobians was less than 10\% in most cases. The results were very similar for both transformation algorithms, i.e., using limb-adjusted Tb's or original ATMS Tb's but calculating empirical coefficients as a function of EIA. The bias was larger for the ATMS observed Tb's than simulated Tb's which is due to error in the radiosonde and ATMS observations, sampling error due to the spatial and temporal differences between the collocated data from the ATMS and radiosonde profiles, as well as error in the RT calculations. These errors either do not exist or will be canceled out when we use the ATMS simulated Tb's to validate the method. Since there is a large interest in calculating LAH for a fixed layer, we also calculated the empirical coefficients using fixed Jacobians for each channel. Generally, the difference between the estimated and calculated LAH values is larger when fixed Jacobians are used. Finally, we investigated the spatial distribution of the bias using two months of ERA-interim data collocated with the ATMS observations. The bias did not indicate any significant regional dependency for the actual Jacobians, but the bias generally increases towards the poles for the fixed Jacobians. Besides, the dependency was larger for the channels sensitive to the lower troposphere than for the channels sensitive to middle and upper troposphere.

\section{APPENDIX}

Earth incidence angle also known as local zenith angle or satellite zenith angle is defined as the angle between the satellite antenna boresight direction (D) and normal to the Earth's surface at the spot location $(\tilde{n})$ and can be mathematically calculated as follows:

$$
\cos \theta=\frac{-D \cdot \tilde{n}}{\|n\|\|D\|}
$$

In a geocentric coordinate system, $\theta$ can be calculated as follows:

$$
\sin \theta=\frac{(h+R) \sin \alpha}{R}
$$

where $\alpha$ is scan angle, $\alpha=0.5 \beta+(f-1) \times \beta, \beta$ is step angle and is equal to $1.111^{\circ}$ for ATMS water vapor channels, $f$ is the ATMS spot number that ranges from 1 for sub-nadir to 48 for the edges of the scan, $\mathrm{R}$ is earth radius $(6371 \mathrm{~km})$, and $\mathrm{h}$ is satellite height $(824 \mathrm{~km})$.

\section{ACKNOWLEDGMENTS}

The views, opinions, and findings contained in this report are those of the authors and should not be construed as an official National Oceanic and Atmospheric Administration or U.S. Government position, policy, or decision. This study was supported by NOAA/NCDC grant\# NA09NES4400006 (Cooperative Institute for Climate and Satellites - CICS) at the University of Maryland, Earth System Science Interdisciplinary Center (ESSIC). 


\section{REFERENCES}

[1] S. Sherwood, "Direct versus indirect effects of tropospheric humidity changes on the hydrologic cycle," Environ. Res. Lett., vol. 5, no. 2, 2010.

[2] I. Held and B. Soden, "Water vapor feedback and global warming," Ann. Rev. Energy Environ., vol. 25, pp. 441-475, 2000.

[3] S. C. Sherwood, R. Roca, T. M. Weckwerth, and N. G. Andronova, "Tropospheric water vapor, convection, and climate," Reviews of Geophysics, vol. 48, no. 2, p. RG2001, Apr. 2010.

[4] K. Minschwaner and A. E. Dessler, "Water vapor feedback in the tropical upper troposphere: Model results and observations," J. Climate, vol. 17, no. 6, pp. 1272-1282, 2004.

[5] B. J. Soden, D. J. Jackson, V. Ramaswamy, M. D. Schwarzkopf, and X. Huang, "The radiative signature of upper tropospheric moistening," Science, vol. 310, no. 5749, pp. 841-844, November 2005.

[6] I. Moradi, S. Buehler, V. John, A. Reale, and R. Ferraro, "Evaluating instrumental inhomogeneities in global radiosonde upper tropospheric humidity data using microwave satellite data," IEEE Trans. Geosci. Remote Sens., vol. 51, no. 6, pp. 3615-3624, 2013.

[7] I. Moradi, B. Soden, R. Ferraro, P. Arkin, and H. Vomel, "Assessing the quality of humidity measurements from global operational radiosonde sensors," J. Geophys. Res., vol. 118, no. 14, pp. 8040-8053, 2013.

[8] W. P. Elliott and D. J. Gaffen, "On the utility of radiosonde humidity archives for climate studies," Bull. Amer. Met. Soc., vol. 72, no. 10, pp. 1507-1520, Oct. 1991.

[9] L. M. Miloshevich, H. Vömel, A. Paukkunen, A. J. Heymsfield, and S. J. Oltmans, "Characterization and correction of relative humidity measurements from Vaisala RS80-A radiosondes at cold temperatures,” J. Atmos. Oceanic Technol., vol. 18, no. 2, pp. 135-156, 2001.

[10] B. J. Soden and F. P. Bretherton, "Upper tropospheric relative humidity from the GOES $6.7 \mu \mathrm{m}$ channel: Method and climatology for july 1987,” J. Geophys. Res., vol. 98, no. D9, 1993.

[11] R. W. Spencer and W. D. Braswell, "How dry is the tropical free troposphere? implications for global warming theory," Bull. Amer. Met. Soc., vol. 78, no. 6, pp. 1097-1106, Jun. 1997.

[12] S. A. Buehler and V. O. John, "A simple method to relate microwave radiances to upper tropospheric humidity," J. Geophys. Res., vol. 110, 2005.

[13] C. Muth, P. Lee, J. Shiue, and W. Webb, "Advanced technology microwave sounder on NPOESS and NPP," in International Geoscience and Remote Sensing Symposium (IGARSS), vol. 4, 2004, pp. 2454-2458.

[14] W. Blackwell, L. Chidester, E. Kim, R. Leslie, C.-H. Lyu, and T. Mo, "NPP ATMS prelaunch performance assessment and sensor data record validation," in Geoscience and Remote Sensing Symposium (IGARSS), 2011 IEEE International, Jul. 2011, pp. 32 -34.

[15] E. E. Borbas, S. W. Seemann, H. Huang, J. Li, and W. Menzel, "Global profile training database for satellite regression retrievals with estimates of skin temperature and emissivity," in Proceedings of the XIV. International ATOVS Study Conference, Beijing, China, 2005.

[16] F. Chevallier, S. Michele, and A. McNally, "Diverse profile datasets from the ecmwf 91-level short-range forecast," NWP SAF Rep. NWPSAF-EC-TR-010, Tech. Rep., 2006, available online at http://research.metoffice.gov.uk/research/interproj/nwpsaf/rtm/profiles_91L.pdf.

[17] G. M. Stokes and S. E. Schwartz, "The atmospheric radiation measurement (ARM) program: Programmatic background and design of the cloud and radiation test bed,” Bull. Amer. Met. Soc., vol. 75, no. 7, pp. 1201-1221, 1994.

[18] L. Garand, D. S. Turner, M. Larocque, J. Bates, S. Boukabara, P. Brunel, F. Chevallier, G. Deblonde, R. Engelen, M. Hollingshead, D. Jackson, G. Jedlovec, J. Joiner, T. Kleespies, D. S. McKague, L. McMillin, J.-L. Moncet, J. R. Pardo, P. J. Rayer, E. Salathe, R. Saunders, N. A. Scott, P. V. Delst, and H. Woolf, "Radiance and Jacobian intercomparison of radiative transfer models applied to HIRS and AMSU channels," J. Geophys. Res., vol. 106, no. D20, pp. 24017-24,031, Oct. 2001.

[19] R. Ferraro, C. Peters-Lidard, C. Hernandez, F. Turk, F. Aires, C. Prigent, X. Lin, S. Boukabara, F. Furuzawa, K. Gopalan, K. Harrison, F. Karbou, L. Li, C. Liu, H. Masunaga, L. Moy, S. Ringerud, G. Skofronick-Jackson, Y. Tian, and N.-Y. Wang, "An evaluation of microwave land surface emissivities over the continental united states to benefit GPM-Era precipitation algorithms," IEEE Trans. Geosci. Remote Sens., vol. 51, no. 1, pp. 378-398, 2013.

[20] R. R. Ferraro, F. Weng, N. C. Grody, L. Zhao, H. Meng, C. Kongoli, P. Pellegrino, S. Qiu, and C. Dean, "NOAA operational hydrological products derived from the advanced microwave sounding unit," IEEE Trans. Geosci. Remote Sens., vol. 43, no. 5, pp. 1036-1049, 2005.

[21] E.-S. Chung, B. Soden, and V. John, "Intercalibrating microwave satellite observations for monitoring long-term variations in upper- and midtropospheric water vapor," J. Atmos. Oceanic Technol., vol. 30, no. 10, pp. 2303-2319, 2013. 
[22] P. Eriksson, S. Buehler, C. Davis, C. Emde, and O. Lemke, “ARTS, the atmospheric radiative transfer simulator, version 2," J. Quant. Spectrosc. Radiat. Transfer, vol. 112, no. 10, pp. 1551 - 1558, 2011.

[23] Q. Liu, P. Van Delst, Y. Chen, D. Groff, Y. Han, A. Collard, F. Weng, S.-A. Boukabara, and J. Derber, "Community radiative transfer model for radiance assimilation and applications," 2012, pp. 3700-3703.

[24] P. W. Rosenkranz, "Water vapor microwave continuum absorption: A comparison of measurements and models," Radio Science, vol. 33, no. 4, pp. 919-928, 1998.

[25] R. Saunders, M. Matricardi, and P. Brunel, "An improved fast radiative transfer model for assimilation of satellite radiance observations," Q. J. R. Meteorol. Soc., vol. 125, no. 556, pp. 1407-1425, 1999.

[26] S. Ding, P. Yang, F. Weng, Q. Liu, Y. Han, P. van Delst, J. Li, and B. Baum, "Validation of the community radiative transfer model," vol. 112, no. 6, pp. 1050-1064, 2011.

[27] B. Burns, X. Wu, and G. Diak, "Effects of precipitation and cloud ice on brightness temperatures in AMSU moisture channels," IEEE Trans. Geosci. Remote Sens., vol. 35, no. 6, pp. 1429-1437, 1997.

[28] S. A. Buehler, M. Kuvatov, T. R. Sreerekha, V. O. John, B. Rydberg, P. Eriksson, and J. Notholt, "A cloud filtering method for microwave upper tropospheric humidity measurements," Atmos. Chem. Phys., vol. 7, no. 21, pp. 5531-5542, 2007.

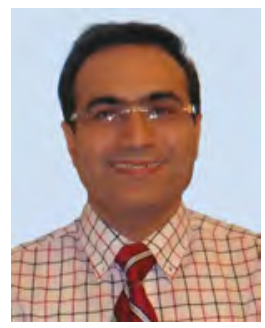

Dr. Isaac Moradi received a M.Sc. in Meteorology from University of Tehran, a Ph.D. in Climatology and Environmental Planning from Kharazmi University of Tehran and a second Ph.D. in Radio and Space Science from Chalmers University of Technology. He is currently working at the Joint Center for Satellite Data Assimilation (JCSDA) and his current activities include satellite data assimilation and developing OSSE data for different practices especially data assimilation, calibration and validation of satellite observations, and microwave radiative transfer modeling. His other research interests include retrieving geophysical variables from microwave satellite data, solar radiation resource assessment, and developing new sensor concepts especially for measuring tropospheric humidity.

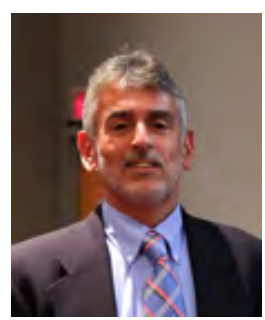

Mr. Ralph R. Ferraro is the Chief of the Satellite Climate Studies Branch (SCSB) of the National Oceanic and Atmospheric Administration (NOAA), National Environmental Satellite, Data and Information Service (NESDIS). His research interests include the use of passive microwave sensors to retrieve parameters related to the hydrological cycle. Mr. Ferraro has earned a B.S. in Meteorology from Cook College Rutgers University and an M.S. in Meteorology from the University of Maryland, College Park.

PLACE

Dr. Brian J. Soden is a Professor at the University of Miami's Rosenstiel School for Marine and Atmospheric Science. Dr. Soden specializes in the use of satellite observations to test and improve computer simulations of Earth's climate.

PHOTO He received his B.S. degree from the University of Miami, and M.S. and Ph.D. degrees from the University of Chicago.

HERE Before returning to the University of Miami, Dr. Soden was a Visiting Scientist and Lecturer at Princeton University, and a Physical Scientist with NOAA's Geophysical Fluid Dynamics Laboratory in Princeton, NJ. Dr. Soden has authored over 100 peer reviewed papers on weather and climate change. He is a Fellow of the American Meteorological Society and Lead Author for the Intergovernmental Panel on Climate Change. Other honors include the American Meteorological Society's Henry G. Houghton Award and the National Space Club's David S. Johnson Award. 


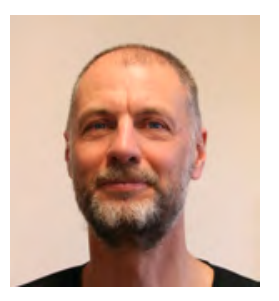

Dr. Patrick Eriksson graduated in engineering physics and the Ph.D. degree in environmental sciences from Chalmers University of Technology, Gothenburg, Sweden, in 1990 and 1999, respectively.

He is currently a Professor of global environmental measurements at the same university. His main areas of expertise are atmospheric radiative transfer and retrieval methodologies, with a focus on passive microwave sounding of the atmosphere. For example, he is a core member of the team around the submillimeter radiometer on board the Odin satellite. He and Stefan Buehler (University of Hamburg) are leading the development of the Atmospheric Radiative

Transfer System (ARTS) open-source software. Based on ARTS, he has implemented a general inversion package

(Qpack), used, for example, by several groups operating ground-based receivers. The present research is directed toward atmospheric water, in both gas and ice phase, between 5 and $100 \mathrm{~km}$. 


\section{LIST OF FIGURES}

1 Jacobians for the ATMS water vapor channels. Radiative transfer calculations were conducted using the LBL model with a surface emissivity of 0.9. The plots are for different atmospheric conditions: (top-left) subarctic winter, (top-right) mid-latitude winter, (bottom-left) subarctic summer, and (bottomright) tropical. The solid lines are for the sub-nadir beam positions and the dashed lines for the edges of the scan. . . . . . . . . . . . . . . . . . . . . . . . . .

Surface contribution for the ATMS water vapor channels as a function of PWV. Ch7.0, Ch4.5, Ch3.0, Ch1.8, and Ch1.0 become insensitive to the surface emissivity when PWV is greater than 30, 20, 10,

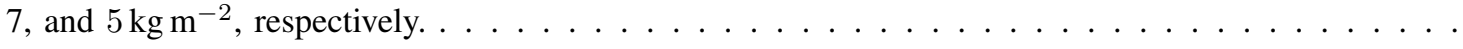
Limb-darkening effect as a function of EIA for the ATMS water vapor channels. The RT simulations were conducted using the LBL model and the combined dataset. . . . . . . . . . . . . . . . . . . Jacobian weighted layer averaged humidity versus simulated satellite Tb's. The columns are for different ATMS channels and the rows show different combinations of datasets and RT models. The first three rows show the results for actual Jacobians and the last row shows the results for the fixed Jacobians. The impact of different datasets and RT models on the estimated LAH values. The values shown are the difference between the LAH values estimated using each dataset and the LAH values estimated using the combined dataset and the LBL model. ALL-FAST indicates the simulations using combined dataset and the fast model and the rest indicates simulations conducted using individual datasets and the LBL model. The markers indicate the datasets and the colors indicate the ATMS channels. . . . . Dependency between empirical coefficients of the transformation method and EIA, (top) coefficient $a$, (bottom) coefficient $b$, and legend shows the ATMS channels. The solid lines indicate the logarithmic fits. The RT simulations were conducted using the combined dataset and the LBL model. . . . . . . . Estimated versus Jacobian weighted LAH values for the ARM radiosonde data. The ATMS brightness temperatures were simulated using the LBL model. The LAH values were calculated using the actual Jacobians for the first two rows and using fixed Jacobians for the last two rows. . . . . . . . . . . . Difference between LAH values calculated using two months of ERA Interim profiles, August 2012 and January 2013, and LAH values estimated using ATMS observations. The rows from top to bottom are for Ch7.0, Ch4.5, Ch3.0, Ch1.8, and Ch1.0. The left column shows the results for actual Jacobians and the right column shows the fixed Jacobians. The collocations are grouped around some specific meridians, because ERA Interim data are available only every 6 hours. . . . . . . . . . . . . Same as Figure 8, but for the difference between ATMS observed Tb's and Tb's simulated using ERA-interim profiles, (top) Ch7.0 and (bottom) Ch1.0. . . . . . . . . . . . . . 


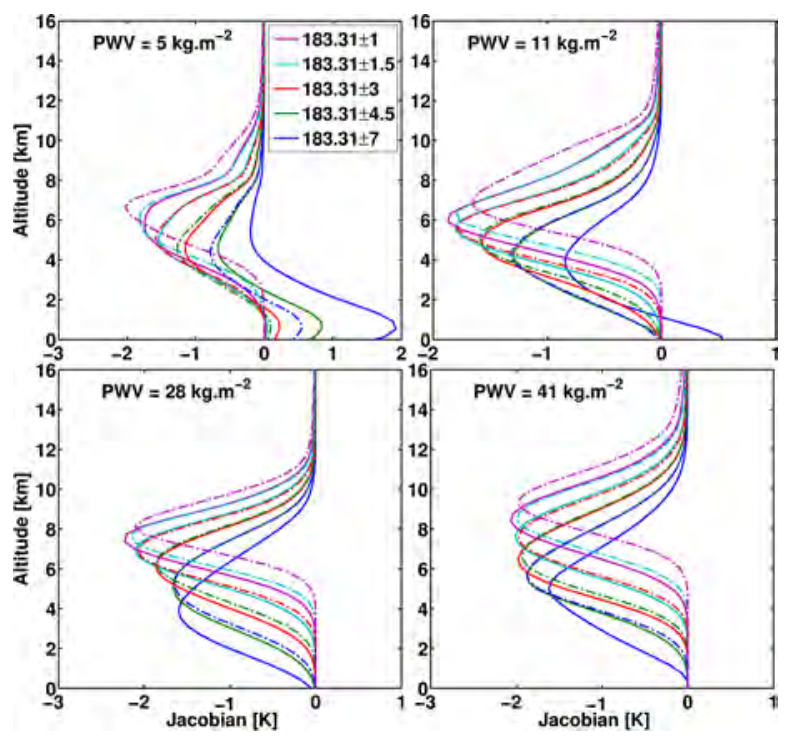

Fig. 1. Jacobians for the ATMS water vapor channels. Radiative transfer calculations were conducted using the LBL model with a surface emissivity of 0.9. The plots are for different atmospheric conditions: (top-left) subarctic winter, (top-right) mid-latitude winter, (bottom-left) subarctic summer, and (bottom-right) tropical. The solid lines are for the sub-nadir beam positions and the dashed lines for the edges of the scan 


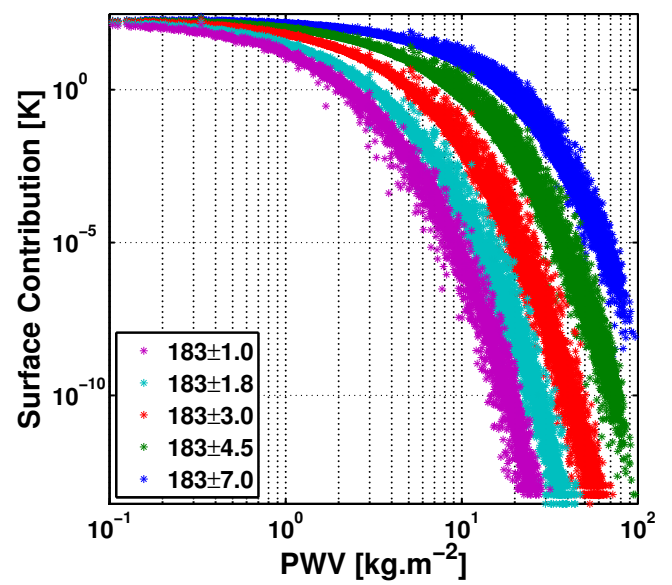

Fig. 2. Surface contribution for the ATMS water vapor channels as a function of PWV. Ch7.0, Ch4.5, Ch3.0, Ch1.8, and Ch1.0 become insensitive to the surface emissivity when PWV is greater than $30,20,10,7$, and $5 \mathrm{~kg} \mathrm{~m}^{-2}$, respectively. 


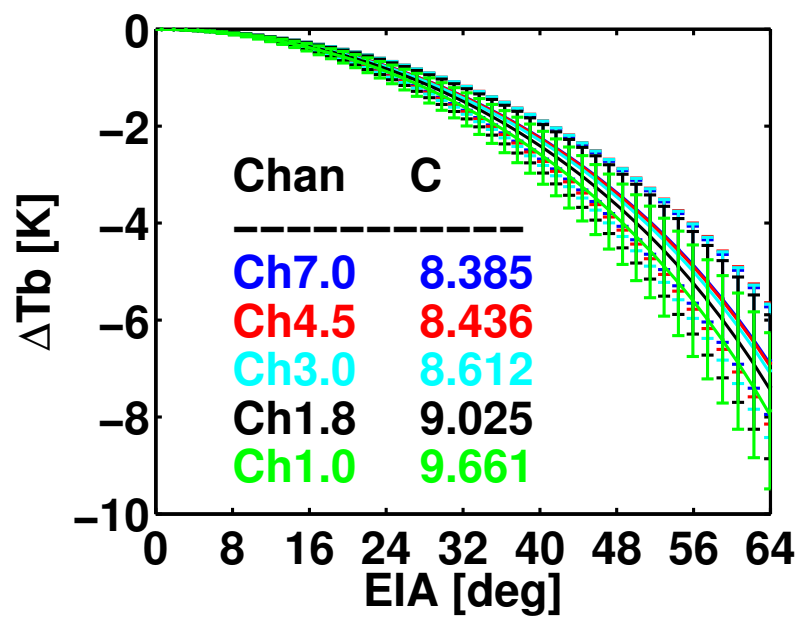

Fig. 3. Limb-darkening effect as a function of EIA for the ATMS water vapor channels. The RT simulations were conducted using the LBL model and the combined dataset. 


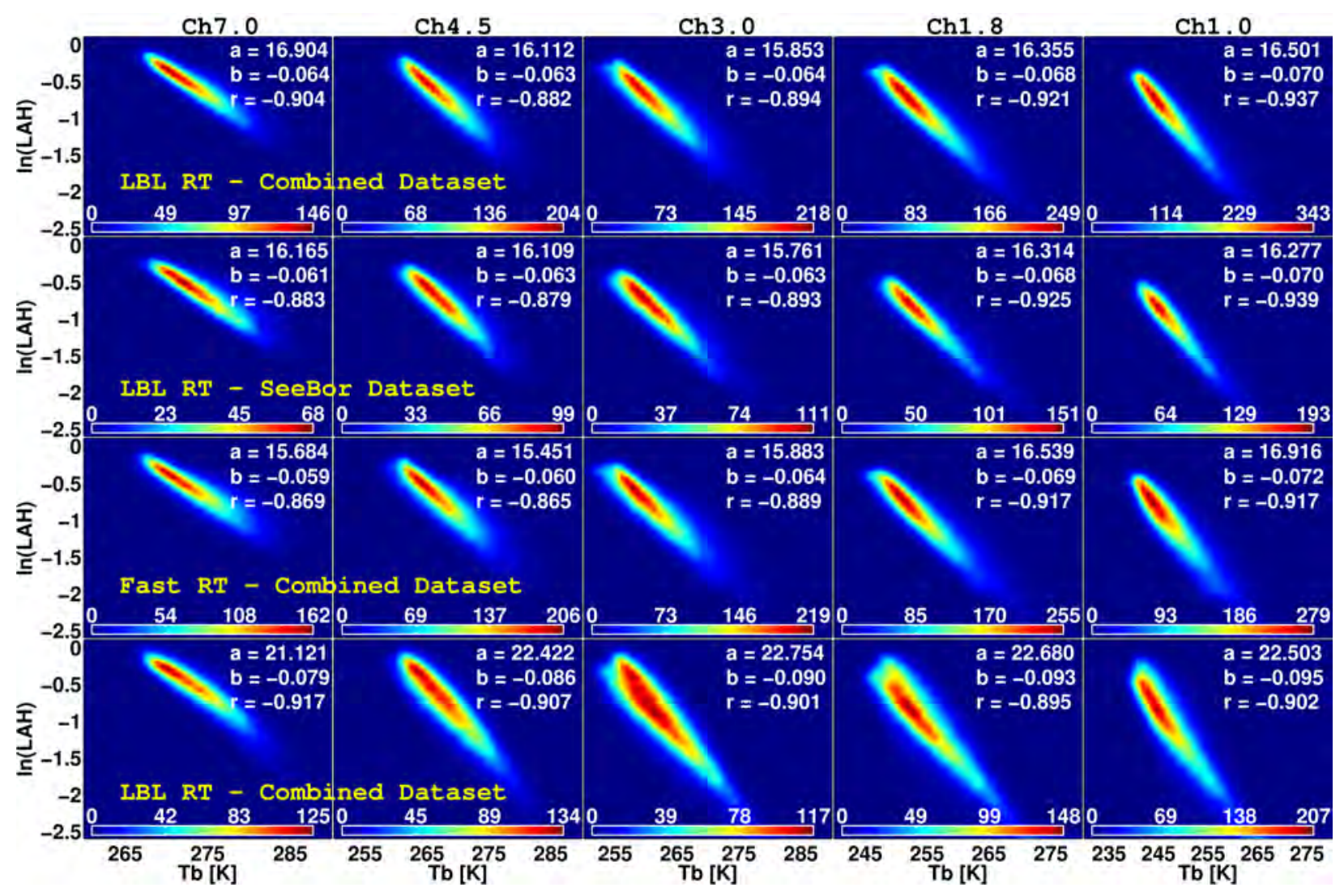

Fig. 4. Jacobian weighted layer averaged humidity versus simulated satellite Tb's. The columns are for different ATMS channels and the rows show different combinations of datasets and RT models. The first three rows show the results for actual Jacobians and the last row shows the results for the fixed Jacobians. 


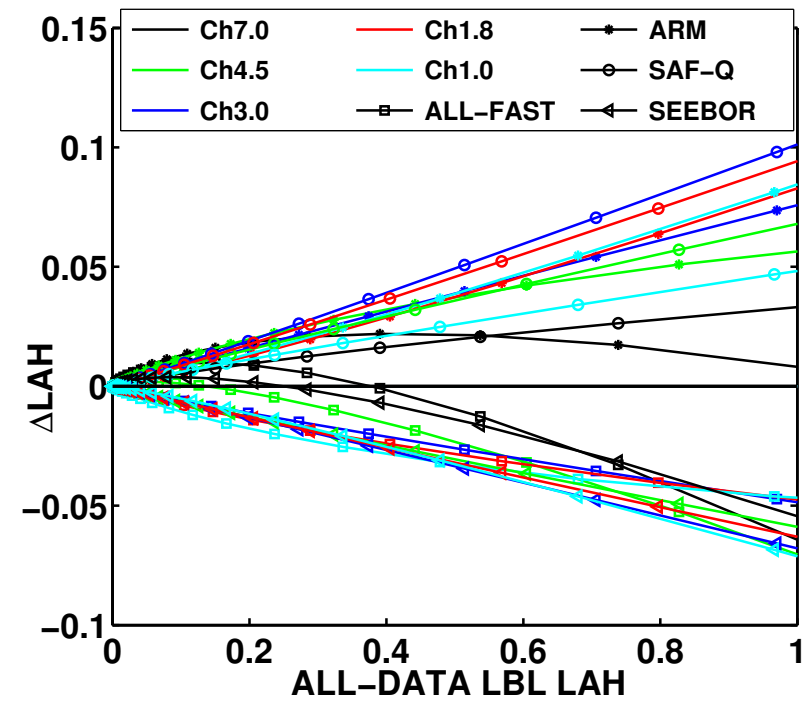

Fig. 5. The impact of different datasets and RT models on the estimated LAH values. The values shown are the difference between the LAH values estimated using each dataset and the LAH values estimated using the combined dataset and the LBL model. ALL-FAST indicates the simulations using combined dataset and the fast model and the rest indicates simulations conducted using individual datasets and the LBL model. The markers indicate the datasets and the colors indicate the ATMS channels. 


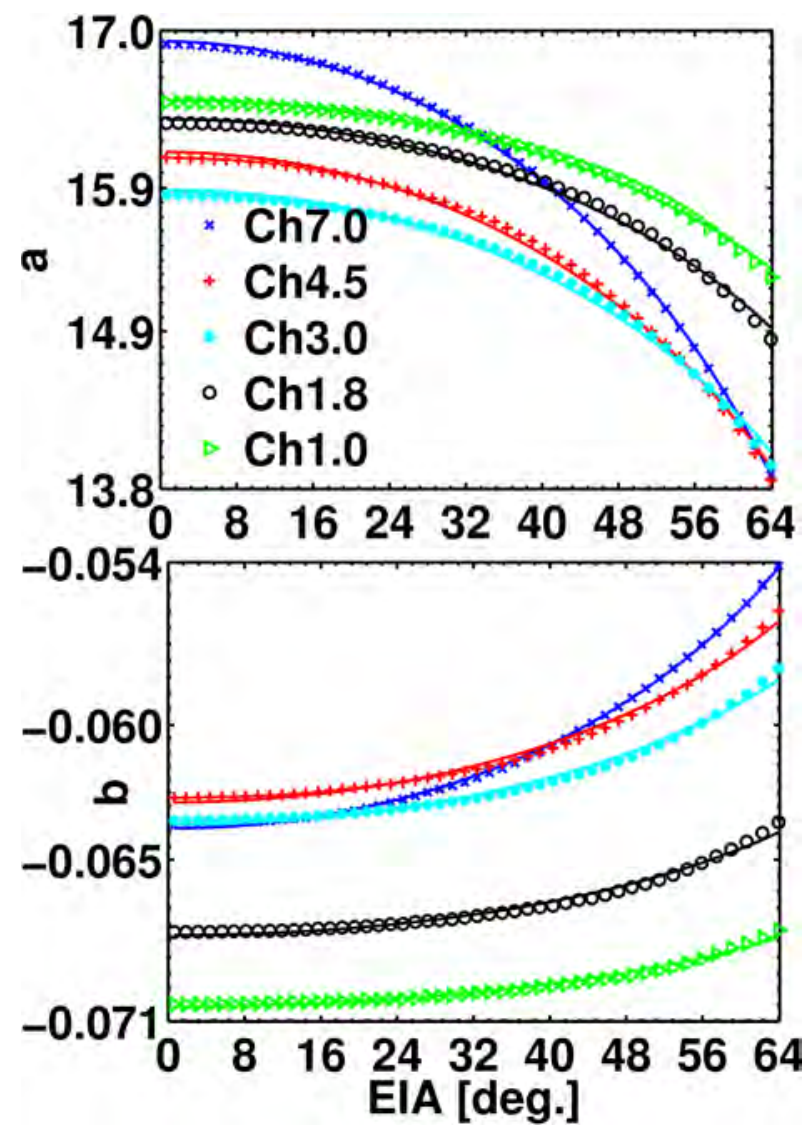

Fig. 6. Dependency between empirical coefficients of the transformation method and EIA, (top) coefficient $a$, (bottom) coefficient $b$, and legend shows the ATMS channels. The solid lines indicate the logarithmic fits. The RT simulations were conducted using the combined dataset and the LBL model. 


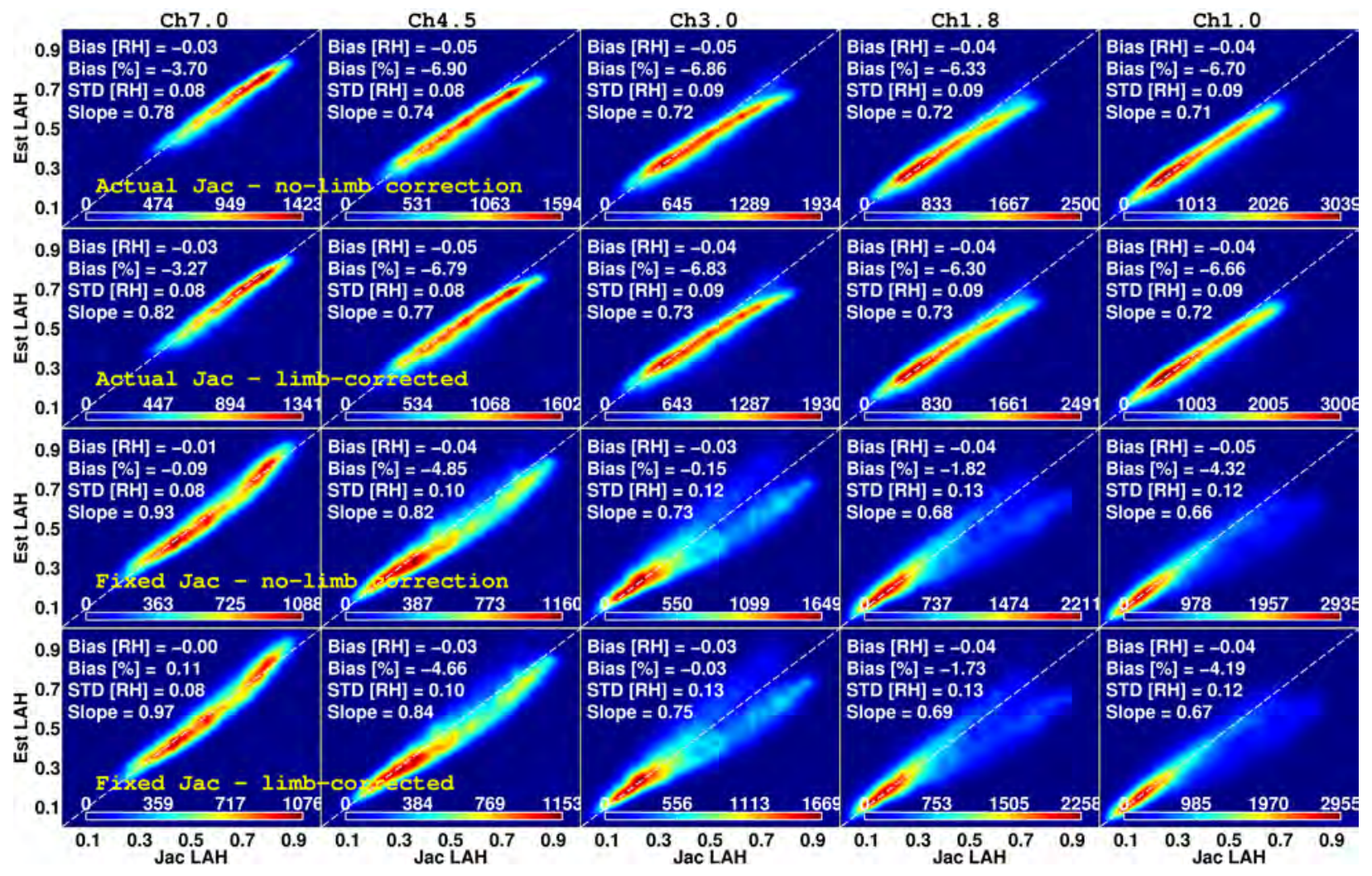

Fig. 7. Estimated versus Jacobian weighted LAH values for the ARM radiosonde data. The ATMS brightness temperatures were simulated using the LBL model. The LAH values were calculated using the actual Jacobians for the first two rows and using fixed Jacobians for the last two rows. 

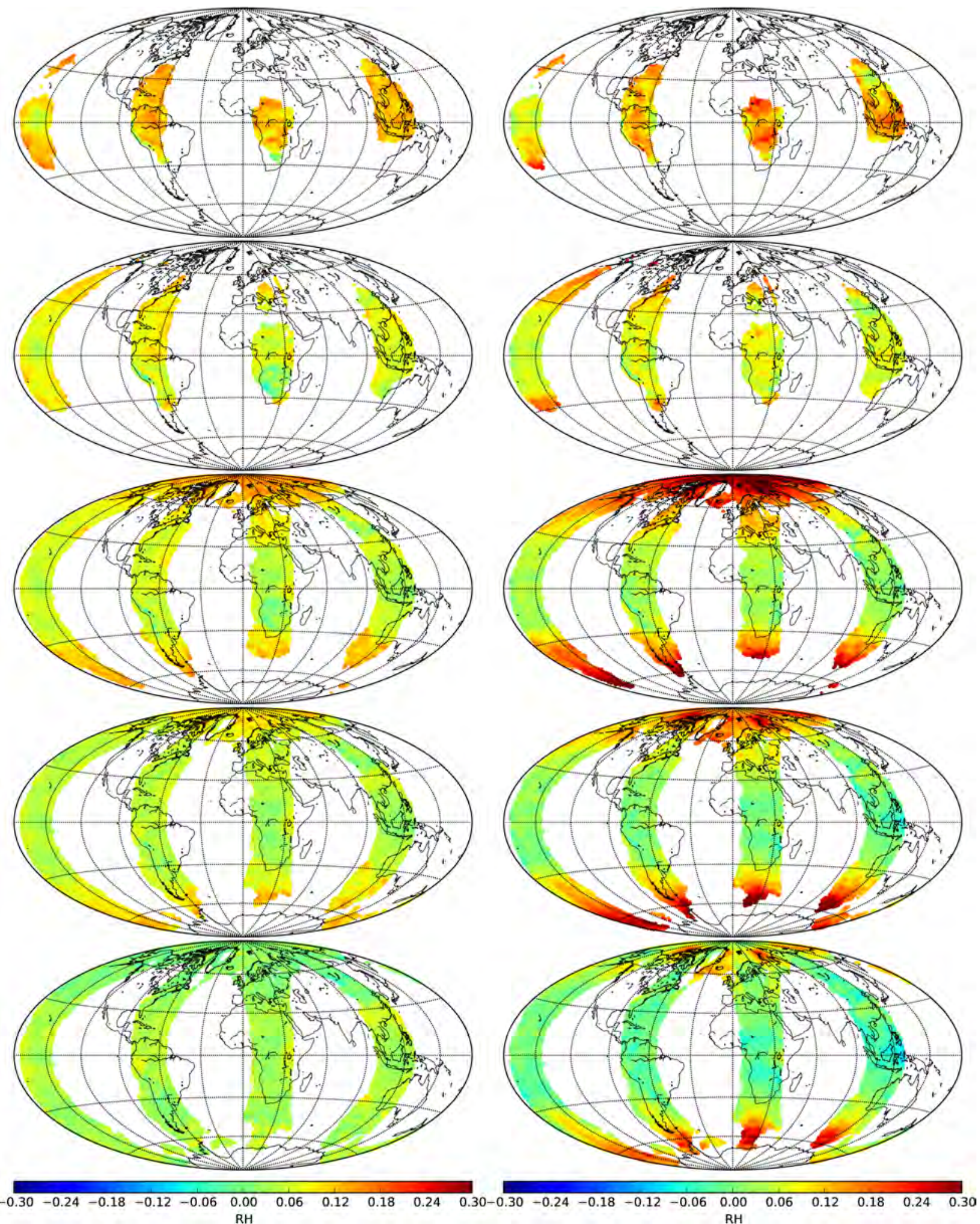

Fig. 8. Difference between LAH values calculated using two months of ERA Interim profiles, August 2012 and January 2013, and LAH values estimated using ATMS observations. The rows from top to bottom are for Ch7.0, Ch4.5, Ch3.0, Ch1.8, and Ch1.0. The left column shows the results for actual Jacobians and the right column shows the fixed Jacobians. The collocations are grouped around some specific meridians, because ERA Interim data are available only every 6 hours. 

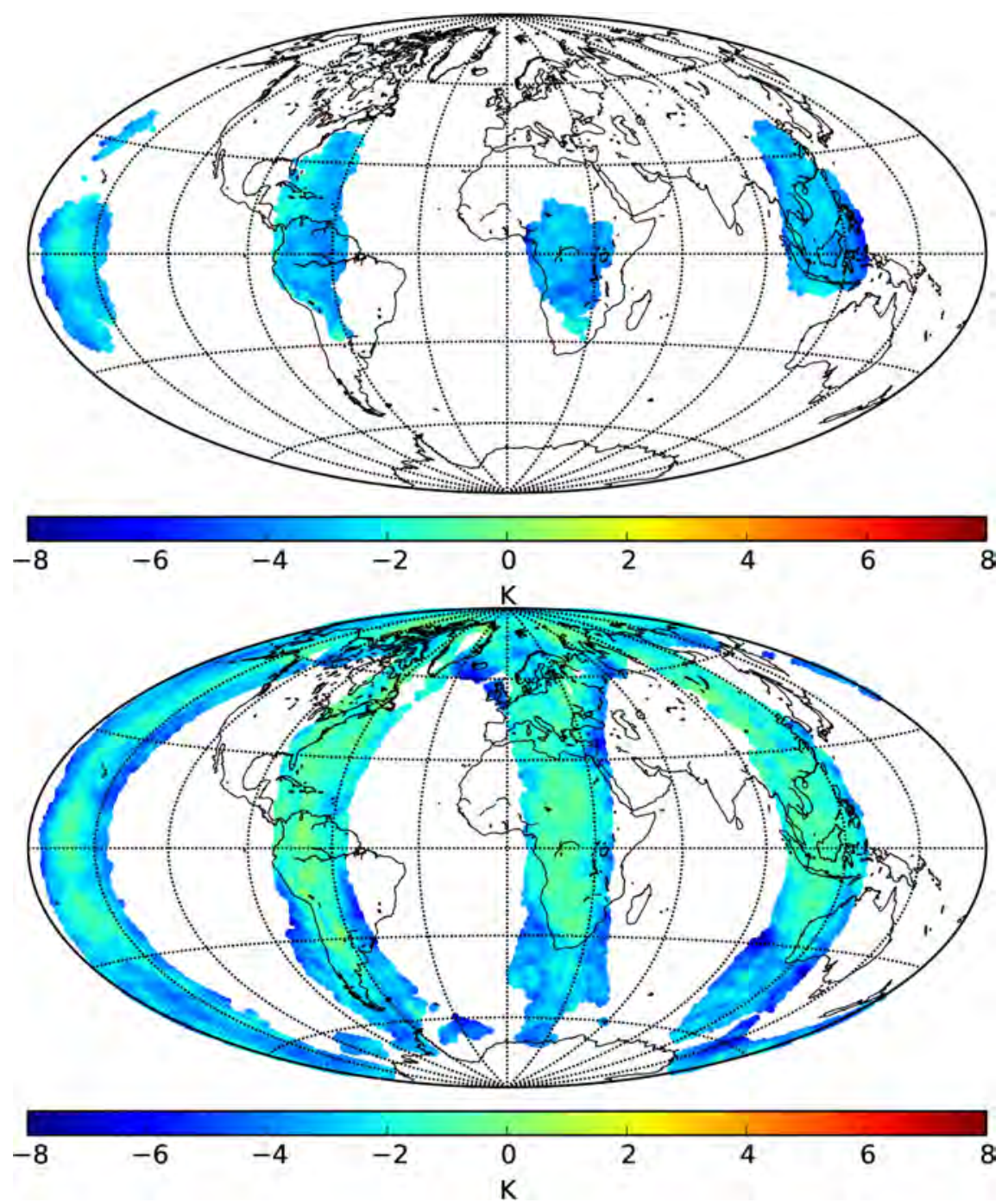

Fig. 9. Same as Figure 8, but for the difference between ATMS observed Tb's and Tb's simulated using ERA-interim profiles, (top) Ch7.0 and (bottom) Ch1.0. 


\section{LIST OF TABLES}

I Coefficients for the limb correction algorithm $(\Delta T b=c \times \ln (\cos \theta))$ calculated using different datasets and RT models. First group indicates the coefficients calculated using the LBL model and the second group using the fast RT model. . . . . . . . . . . . . . . . . . . . . . . . . . . . 29

II Coefficients for the logarithmic relations, $p=p_{1}+p_{2} \times \ln (\cos \theta)$, calculated using the LBL model and combined dataset: (top) actual Jacobians, (bottom) fixed Jacobians. The last two columns indicate

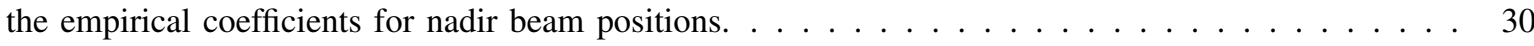

III Statistics for the estimated versus Jacobian weighted LAH. STD is standard deviation of the differences between estimated and calculated values. . . . . . . . . . . . . . . .

IV Statistics for the differences between the LAH values estimated from the ATMS observations and the LAH values calculated using the ARM radiosonde profiles. Jacobians were calculated using the LBL model. The AJ and FJ stand for actual and fixed Jacobians, respectively, CA and TLA indicate the results for coefficient adjustment and Tb limb adjustment, respectively, STD is standard deviation of the differences between estimated and calculated LAH, and Bias Tb refers to the difference between the observed and simulated brightness temperatures. NSA-C1 did not have enough collocations for

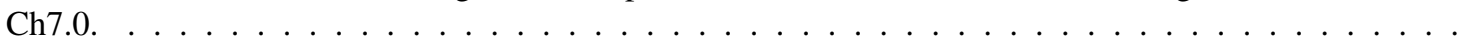

V Same as Table IV but the ATMS observed Tb's were adjusted for the differences between the simulated

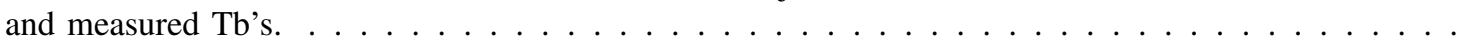


TABLE I

COEFFICIENTS FOR THE LIMB CORRECTION ALGORITHM $(\Delta T b=c \times \ln (\cos \theta))$ CALCULATED USING DIFFERENT DATASETS AND RT MODELS. FIRST GROUP INDICATES THE COEFFICIENTS CALCULATED USING THE LBL MODEL AND THE SECOND GROUP USING THE FAST RT MODEL.

\begin{tabular}{llllll}
\hline Chan & Ch7.0 & Ch4.5 & Ch3.0 & Ch1.8 & Ch1.0 \\
\hline ARM & 8.3739 & 8.2727 & 8.3737 & 8.7616 & 9.3527 \\
SAF-Q & 8.4454 & 8.5252 & 8.6633 & 9.1349 & 9.8526 \\
SAF-T & & 8.0117 & 8.4287 & 8.9495 & 9.6012 \\
SEEBOR & 8.3651 & 8.4821 & 8.7201 & 9.1360 & 9.7764 \\
ALL-DATA & 8.3848 & 8.4361 & 8.6115 & 9.0253 & 9.6609 \\
\hline ARM & 8.8890 & 8.7036 & 8.8209 & 9.0752 & 9.6274 \\
SAF-Q & 8.7686 & 8.8347 & 8.9797 & 9.4149 & 10.0172 \\
SAF-T & & 8.3385 & 8.7007 & 9.1490 & 9.6697 \\
SEEBOR & 8.6572 & 8.8004 & 9.0180 & 9.3964 & 9.9398 \\
ALL-DATA & 8.7385 & 8.7741 & 8.9435 & 9.2935 & 9.8425 \\
\hline
\end{tabular}


TABLE II

COEFFICIENTS FOR THE LOGARITHMIC RELATIONS, $p=p_{1}+p_{2} \times \ln (\cos \theta)$, CALCULATED USING THE LBL MODEL AND COMBINED DATASET: (TOP) ACTUAL JACOBIANS, (BOTTOM) FIXED JACOBIANS. THE LAST TWO COLUMNS INDICATE THE EMPIRICAL COEFFICIENTS FOR NADIR BEAM POSITIONS.

\begin{tabular}{lllllll}
\hline Chan & $a_{1}$ & $a_{2}$ & $b_{1}$ & $b_{2}$ & $a$ & $b$ \\
\hline Ch7.0 & 16.926416 & 3.675071 & -0.063834 & -0.011692 & 16.904 & -0.0641 \\
Ch4.5 & 16.153449 & 2.670072 & -0.062870 & -0.008139 & 16.112 & -0.0631 \\
Ch3.0 & 15.889499 & 2.218520 & -0.063687 & -0.006505 & 15.853 & -0.0641 \\
Ch1.8 & 16.382202 & 1.766304 & -0.067800 & -0.004630 & 16.355 & -0.0681 \\
Ch1.0 & 16.516412 & 1.428877 & -0.070436 & -0.003093 & 16.501 & -0.0700 \\
\hline Ch7.0 & 21.111663 & 4.384297 & -0.079078 & -0.013845 & 21.121 & -0.0791 \\
Ch4.5 & 22.455108 & 3.433289 & -0.086548 & -0.010267 & 22.422 & -0.0861 \\
Ch3.0 & 22.797294 & 2.774498 & -0.090363 & -0.007760 & 22.754 & -0.0901 \\
Ch1.8 & 22.713884 & 2.169595 & -0.093041 & -0.005320 & 22.680 & -0.0931 \\
Ch1.0 & 22.523808 & 1.731349 & -0.095061 & -0.003347 & 22.503 & -0.0950 \\
\hline
\end{tabular}


TABLE III

STATISTICS FOR THE ESTIMATED VERSUS JACOBIAN WEIGHTED LAH. STD IS STANDARD DEVIATION OF THE DIFFERENCES BETWEEN ESTIMATED AND CALCULATED VALUES.

\begin{tabular}{|c|c|c|c|c|c|c|c|c|c|c|c|c|c|c|c|c|c|c|c|c|}
\hline \multirow[b]{2}{*}{ 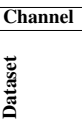 } & \multicolumn{4}{|c|}{ Ch7.0 } & \multicolumn{4}{|c|}{ Ch4.5 } & \multicolumn{4}{|c|}{ Ch3.0 } & \multicolumn{4}{|c|}{ Ch1.8 } & \multicolumn{4}{|c|}{ Ch1.0 } \\
\hline & 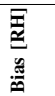 & $\frac{\bar{s}}{\sqrt[5]{50}}$ & 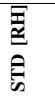 & कू̆ & 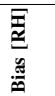 & 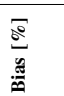 & 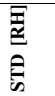 & के & 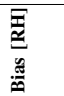 & $\frac{\overline{2}}{2}$ & 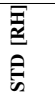 & के & 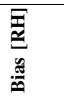 & $\frac{\sqrt{0}}{0}$ & 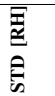 & के & 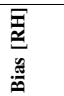 & 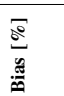 & 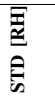 & के \\
\hline \multicolumn{21}{|c|}{ LBL Model - Actual Jacobian - No-limb Correction } \\
\hline ARM & -0.03 & -3.70 & 0.08 & 0.78 & -0.05 & -6.90 & 0.08 & 0.74 & -0.05 & -6.86 & 0.09 & 0.72 & -0.04 & -6.33 & 0.09 & 0.72 & -0.04 & -6.70 & 0.09 & 0.71 \\
\hline SAF-Q & -0.02 & -3.01 & 0.06 & 0.83 & -0.04 & -6.04 & 0.07 & 0.78 & -0.05 & -7.76 & 0.07 & 0.77 & -0.04 & -7.22 & 0.06 & 0.80 & -0.03 & -3.94 & 0.05 & 0.83 \\
\hline SAF-T & 0.01 & 1.98 & 0.06 & 1.00 & 0.00 & 1.24 & 0.07 & 0.97 & 0.02 & 4.99 & 0.06 & 0.95 & 0.00 & 2.55 & 0.06 & 0.91 & -0.02 & -2.53 & 0.06 & 0.86 \\
\hline SEEBOR & 0.01 & 4.12 & 0.07 & 0.84 & 0.02 & 7.72 & 0.07 & 0.81 & 0.02 & 9.01 & 0.07 & 0.83 & 0.02 & 8.22 & 0.06 & 0.87 & 0.02 & 7.86 & 0.05 & 0.90 \\
\hline \multicolumn{21}{|c|}{ LBL Model - Fixed Jacobians - No-limb Correction } \\
\hline ARM & -0.01 & -0.09 & 0.08 & 0.93 & -0.04 & -4.85 & 0.10 & 0.82 & -0.03 & -0.25 & 0.12 & 0.73 & -0.04 & -1.96 & 0.13 & 0.68 & -0.05 & -4.56 & 0.12 & 0.66 \\
\hline SAF-Q & -0.03 & -4.21 & 0.10 & 0.81 & -0.05 & -6.81 & 0.11 & 0.73 & -0.07 & -8.74 & 0.12 & 0.67 & -0.07 & -9.04 & 0.13 & 0.62 & -0.05 & -4.31 & 0.13 & 0.62 \\
\hline SAF-T & 0.02 & 1.29 & 0.10 & 1.13 & 0.04 & 7.47 & 0.10 & 1.06 & 0.09 & 24.70 & 0.11 & 1.04 & 0.06 & 23.05 & 0.09 & 0.94 & 0.03 & 18.46 & 0.08 & 0.86 \\
\hline SEEBOR & 0.03 & 9.09 & 0.07 & 0.89 & 0.02 & 9.00 & 0.09 & 0.85 & 0.02 & 9.89 & 0.10 & 0.82 & 0.01 & 7.90 & 0.09 & 0.79 & 0.00 & 7.57 & 0.09 & 0.80 \\
\hline \multicolumn{21}{|c|}{ LBL Model - Actual Jacobians - Limb-corrted Data } \\
\hline ARM & -0.03 & -3.27 & 0.08 & 0.82 & -0.05 & -6.79 & 0.08 & 0.77 & -0.04 & -6.83 & 0.09 & 0.73 & -0.04 & -6.30 & 0.09 & 0.73 & -0.04 & -6.66 & 0.09 & 0.72 \\
\hline SAF-Q & -0.02 & -2.88 & 0.07 & 0.86 & -0.04 & -5.93 & 0.07 & 0.80 & -0.05 & -7.73 & 0.07 & 0.79 & -0.04 & -7.16 & 0.06 & 0.81 & -0.02 & -3.82 & 0.05 & 0.84 \\
\hline SAF-T & 0.02 & 2.88 & 0.06 & 1.05 & 0.01 & 1.83 & 0.07 & 1.00 & 0.02 & 5.54 & 0.07 & 0.97 & 0.01 & 2.90 & 0.06 & 0.92 & -0.02 & -2.32 & 0.06 & 0.87 \\
\hline SEEBOR & 0.01 & 3.96 & 0.07 & 0.87 & 0.02 & 7.76 & 0.07 & 0.83 & 0.03 & 9.01 & 0.07 & 0.85 & 0.02 & 8.24 & 0.06 & 0.89 & 0.02 & 7.92 & 0.05 & 0.91 \\
\hline \multicolumn{21}{|c|}{ LBL Model - Fixed Jacobians - Limb-corrected Data } \\
\hline ARM & -0.00 & 0.11 & 0.08 & 0.97 & -0.03 & -4.66 & 0.10 & 0.8 & -0.03 & -0.13 & 0.13 & 0.75 & -0.04 & -1.87 & 0.13 & 0.69 & -0.04 & -4.43 & 0.12 & 0.67 \\
\hline SAF-Q & -0.03 & -3.99 & 0.11 & 0.84 & -0.05 & -6.61 & 0.11 & 0.75 & -0.07 & -8.65 & 0.12 & 0.68 & -0.07 & -8.90 & 0.13 & 0.63 & -0.05 & -4.08 & 0.13 & 0.62 \\
\hline SAF-T & 0.03 & 2.44 & 0.11 & 1.19 & 0.05 & 8.28 & 0.10 & 1.09 & 0.10 & 25.49 & 0.11 & 1.07 & 0.07 & 23.63 & 0.09 & 0.95 & 0.03 & 18.82 & 0.08 & 0.87 \\
\hline SEEBOR & 0.03 & 8.81 & 0.07 & 0.93 & 0.02 & 9.02 & 0.09 & 0.88 & 0.02 & 9.96 & 0.10 & 0.84 & 0.01 & 8.01 & 0.10 & 0.80 & 0.01 & 7.74 & 0.09 & 0.80 \\
\hline \multicolumn{21}{|c|}{ Fast Model - Actual Jacobians - No-limb Correction } \\
\hline ARM & -0.01 & -1.78 & 0.10 & 1.03 & -0.02 & -3.46 & 0.11 & 0. & -0.02 & -2.0 & 0.06 & 0. & -0.01 & -0.47 & 0.10 & 0.94 & -0.01 & 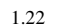 & 0.08 & 0.85 \\
\hline SAF-Q & -0.01 & -1.43 & 0.07 & 0.84 & -0.03 & -3.08 & 0.07 & 0.79 & -0.03 & -3.13 & 0.07 & 0.79 & -0.01 & 1.23 & 0.06 & 0.81 & 0.02 & 11.95 & 0.07 & 0.81 \\
\hline SAF-T & 0.02 & 3.39 & 0.07 & 1.01 & 0.02 & 3.62 & 0.07 & 0.99 & 0.03 & 7.82 & 0.06 & 0.97 & 0.01 & 4.48 & 0.06 & 0.92 & -0.02 & -2.16 & 0.06 & 0.85 \\
\hline SEEBOR & 0.03 & 7.37 & 0.07 & 0.85 & 0.04 & 12.06 & 0.07 & 0.83 & 0.04 & 15.11 & 0.07 & 0.86 & 0.04 & 15.60 & 0.06 & 0.90 & 0.04 & 18.10 & 0.07 & 0.88 \\
\hline \multicolumn{21}{|c|}{ Fast Model - Actual Jacobians - Limb-corrected Data } \\
\hline ARM & -0.01 & -1.60 & 0.10 & 1.07 & -0.02 & -3.42 & 0.10 & 0.96 & -0.02 & -2.00 & 0.06 & 0.85 & -0.01 & -0.41 & 0.10 & 0.95 & -0.01 & 1.27 & 0.08 & 0.86 \\
\hline SAF-Q & -0.01 & -1.23 & 0.07 & 0.88 & -0.02 & -2.93 & 0.07 & 0.82 & -0.03 & -3.09 & 0.07 & 0.80 & -0.01 & 1.29 & 0.06 & 0.82 & 0.02 & 12.06 & 0.07 & 0.81 \\
\hline SAF-T & 0.03 & 4.30 & 0.07 & 1.06 & 0.02 & 4.22 & 0.07 & 1.02 & 0.04 & 8.35 & 0.07 & 0.99 & 0.01 & 4.82 & 0.06 & 0.93 & -0.02 & -1.98 & 0.06 & 0.86 \\
\hline SEEBOR & 0.03 & 7.27 & 0.07 & 0.89 & 0.04 & 12.10 & 0.07 & 0.85 & 0.04 & 15.11 & 0.07 & 0.88 & 0.04 & 15.63 & 0.06 & 0.91 & 0.04 & 18.16 & 0.07 & 0.89 \\
\hline
\end{tabular}


TABLE IV

STATISTICS FOR THE DIFFERENCES BETWEEN THE LAH VALUES ESTIMATED FROM THE ATMS OBSERVATIONS AND THE LAH VALUES CALCULATED USING THE ARM RADIOSONDE PROFILES. JACOBIANS WERE CALCULATED USING THE LBL MODEL. THE AJ AND FJ STAND FOR ACTUAL AND FIXED JACOBIANS, RESPECTIVELY, CA AND TLA INDICATE THE RESULTS FOR COEFFICIENT ADJUSTMENT AND TB LIMB ADJUSTMENT, RESPECTIVELY, STD IS STANDARD DEVIATION OF THE DIFFERENCES BETWEEN ESTIMATED AND CALCULATED LAH, AND BIAS TB REFERS TO THE DIFFERENCE BETWEEN THE OBSERVED AND SIMULATED BRIGHTNESS TEMPERATURES. NSA-C1 DID NOT HAVE ENOUGH COLLOCATIONS FOR CH7.0.

\begin{tabular}{|c|c|c|c|c|c|c|c|c|c|c|c|c|c|c|c|c|c|c|c|}
\hline & 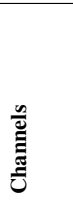 & 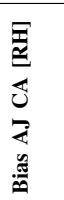 & 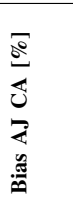 & 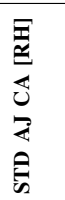 & 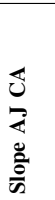 & 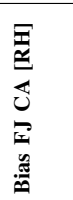 & 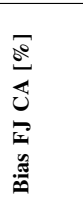 & 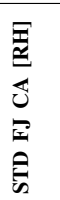 & 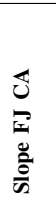 & 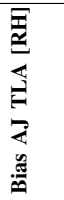 & 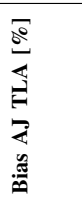 & 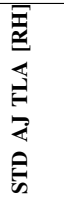 & 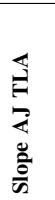 & 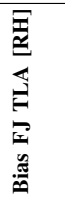 & 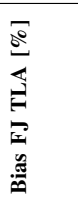 & 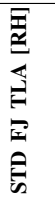 & 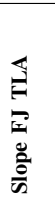 & 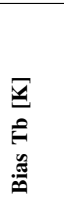 & 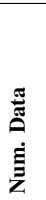 \\
\hline NSA-C1 & Ch4.5 & 0.07 & 9.40 & 0.06 & 0.28 & 0.23 & 35.02 & 0.09 & 0.71 & 0.10 & 13.21 & 0.07 & 0.40 & 0.27 & 40.82 & 0.10 & 0.78 & -1.28 & 78 \\
\hline NSA-C1 & Ch3.0 & 0.07 & 13.43 & 0.07 & 0.63 & 0.21 & 46.64 & 0.10 & 0.84 & 0.08 & 15.93 & 0.08 & 0.67 & 0.23 & 50.83 & 0.11 & 0.89 & -1.49 & 290 \\
\hline NSA-C1 & Ch1.8 & 0.05 & 12.76 & 0.08 & 0.59 & 0.13 & 35.12 & 0.10 & 0.72 & 0.06 & 14.34 & 0.08 & 0.61 & 0.15 & 37.70 & 0.10 & 0.75 & -1.44 & 346 \\
\hline NSA-C1 & Ch1.0 & -0.01 & 1.89 & 0.08 & 0.50 & 0.03 & 13.11 & 0.09 & 0.59 & -0.01 & 2.59 & 0.08 & 0.51 & 0.04 & 14.20 & 0.09 & 0.61 & -0.83 & 351 \\
\hline SGP-C1 & Ch7.0 & 0.01 & 3.46 & 0.07 & 0.73 & 0.07 & 19.78 & 0.06 & 0.92 & 0.02 & 4.85 & 0.08 & 0.78 & 0.08 & 19.89 & 0.07 & 0.99 & -1.53 & 128 \\
\hline SGP-C1 & Ch4.5 & 0.01 & 7.50 & 0.08 & 0.72 & 0.08 & 27.51 & 0.08 & 0.93 & 0.02 & 7.47 & 0.08 & 0.76 & 0.08 & 28.11 & 0.09 & 0.99 & -1.49 & 270 \\
\hline SGP-C1 & Ch3.0 & 0.02 & 11.14 & 0.09 & 0.70 & 0.09 & 37.07 & 0.09 & 0.94 & 0.02 & 11.19 & 0.09 & 0.73 & 0.09 & 37.96 & 0.10 & 0.98 & -1.77 & 451 \\
\hline SGP-C1 & Ch1.8 & 0.02 & 12.78 & 0.09 & 0.71 & 0.07 & 35.01 & 0.09 & 0.86 & 0.02 & 12.97 & 0.09 & 0.73 & 0.07 & 35.69 & 0.09 & 0.89 & -1.86 & 493 \\
\hline SGP-C1 & Ch1.0 & 0.01 & 10.49 & 0.09 & 0.65 & 0.04 & 29.23 & 0.09 & 0.75 & 0.01 & 10.58 & 0.09 & 0.66 & 0.04 & 29.73 & 0.09 & 0.76 & -1.70 & 533 \\
\hline TWP-C1 & Ch7.0 & 0.06 & 10.71 & 0.06 & 0.92 & 0.12 & 19.02 & 0.09 & 1.03 & 0.08 & 13.32 & 0.07 & 1.02 & 0.15 & 22.95 & 0.11 & 1.15 & -1.83 & 312 \\
\hline TWP-C1 & Ch4.5 & 0.00 & 2.24 & 0.06 & 0.77 & 0.02 & 5.59 & 0.09 & 0.89 & 0.01 & 3.59 & 0.06 & 0.83 & 0.04 & 7.82 & 0.09 & 0.95 & -1.24 & 312 \\
\hline TWP-C1 & Ch3.0 & -0.03 & -2.31 & 0.07 & 0.69 & -0.04 & -3.21 & 0.10 & 0.74 & -0.02 & -1.53 & 0.07 & 0.73 & -0.03 & -2.01 & 0.10 & 0.78 & -1.10 & 312 \\
\hline TWP-C1 & Ch1.8 & -0.04 & -4.48 & 0.07 & 0.67 & -0.07 & -8.17 & 0.11 & 0.63 & -0.03 & -3.97 & 0.07 & 0.70 & -0.07 & -7.44 & 0.11 & 0.65 & -0.88 & 312 \\
\hline TWP-C1 & Ch1.0 & -0.04 & -5.96 & 0.07 & 0.64 & -0.09 & -10.78 & 0.12 & 0.53 & -0.04 & -5.61 & 0.07 & 0.65 & -0.09 & -10.26 & 0.12 & 0.54 & -0.30 & 312 \\
\hline TWP-C2 & Ch7.0 & 0.06 & 12.45 & 0.06 & 0.85 & 0.10 & 20.08 & 0.07 & 0.94 & 0.07 & 13.52 & 0.06 & 0.93 & 0.11 & 21.81 & 0.08 & 1.02 & -1.74 & 430 \\
\hline TWP-C2 & Ch4.5 & 0.02 & 7.06 & 0.06 & 0.75 & 0.03 & 9.42 & 0.07 & 0.83 & 0.02 & 7.50 & 0.06 & 0.79 & 0.04 & 10.50 & 0.08 & 0.88 & -1.32 & 436 \\
\hline TWP-C2 & Ch3.0 & 0.00 & 4.06 & 0.06 & 0.71 & 0.00 & 4.28 & 0.08 & 0.74 & 0.01 & 4.20 & 0.06 & 0.74 & 0.00 & 4.87 & 0.08 & 0.77 & -1.37 & 436 \\
\hline TWP-C2 & Ch1.8 & -0.00 & 2.96 & 0.06 & 0.71 & -0.01 & 5.66 & 0.09 & 0.66 & -0.00 & 3.11 & 0.06 & 0.73 & -0.00 & 6.12 & 0.09 & 0.67 & -1.44 & 436 \\
\hline TWP-C2 & Ch1.0 & -0.01 & 2.07 & 0.06 & 0.69 & -0.00 & 10.17 & 0.10 & 0.58 & -0.01 & 2.22 & 0.06 & 0.70 & -0.00 & 10.62 & 0.10 & 0.59 & -1.10 & 436 \\
\hline TWP-C3 & Ch7.0 & 0.38 & 87.13 & 0.15 & 0.79 & 0.70 & 157.07 & 0.21 & 0.98 & 0.61 & 139.67 & 0.21 & 0.84 & 1.21 & 269.58 & 0.32 & 1.08 & -3.34 & 28 \\
\hline TWP-C3 & Ch4.5 & 0.26 & 67.42 & 0.12 & 0.73 & 0.63 & 161.24 & 0.19 & 0.84 & 0.36 & 92.84 & 0.14 & 0.78 & 0.95 & 238.32 & 0.25 & 0.92 & -2.91 & 35 \\
\hline TWP-C3 & Ch3.0 & 0.20 & 60.09 & 0.11 & 0.69 & 0.52 & 150.91 & 0.17 & 0.73 & 0.26 & 74.60 & 0.12 & 0.74 & 0.69 & 197.47 & 0.20 & 0.78 & -3.45 & 39 \\
\hline TWP-C3 & Ch1.8 & 0.17 & 56.64 & 0.10 & 0.69 & 0.33 & 114.35 & 0.15 & 0.63 & 0.20 & 63.82 & 0.11 & 0.72 & 0.40 & 132.60 & 0.16 & 0.66 & -3.91 & 39 \\
\hline TWP-C3 & Ch1.0 & 0.13 & 46.82 & 0.08 & 0.77 & 0.19 & 79.52 & 0.14 & 0.60 & 0.14 & 49.59 & 0.09 & 0.79 & 0.21 & 84.90 & 0.14 & 0.61 & -3.82 & 39 \\
\hline
\end{tabular}


TABLE V

SAME AS TABLE IV BUt THE ATMS OBSERVED TB'S WERE ADJUSTED FOR THE DIFFERENCES BETWEEN THE SIMULATED AND MEASURED Тв's.

\begin{tabular}{|c|c|c|c|c|c|c|c|c|c|c|c|c|c|c|c|c|c|c|c|}
\hline 韋 & & 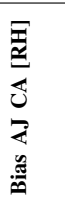 & 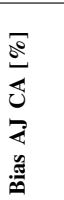 & 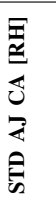 & 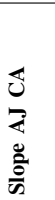 & 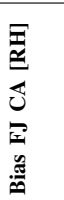 & 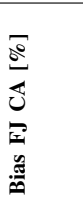 & 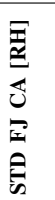 & 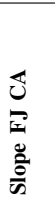 & 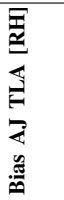 & 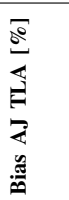 & 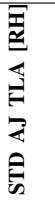 & 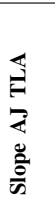 & 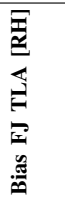 & 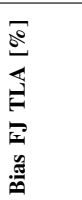 & 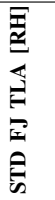 & 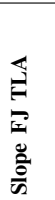 & $\begin{array}{l}\bar{v} \\
\theta \\
0 \\
0 \\
0 \\
0\end{array}$ & 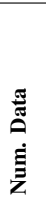 \\
\hline NSA-C1 & Ch4.5 & 0.01 & 1.41 & 0.06 & 0.27 & 0.14 & 21.56 & 0.08 & 0.64 & 0.03 & 4.48 & 0.06 & 0.37 & 0.17 & 26.07 & 0.09 & 0.70 & -1.28 & 78 \\
\hline NSA-C1 & Ch3.0 & 0.01 & 3.63 & 0.07 & 0.57 & 0.12 & 28.89 & 0.10 & 0.74 & 0.02 & 5.46 & 0.08 & 0.61 & 0.14 & 31.87 & 0.10 & 0.78 & -1.49 & 290 \\
\hline NSA-C1 & Ch1.8 & -0.00 & 2.60 & 0.08 & 0.54 & 0.06 & 18.63 & 0.10 & 0.63 & 0.00 & 3.72 & 0.08 & 0.56 & 0.07 & 20.46 & 0.10 & 0.65 & -1.44 & 346 \\
\hline NSA-C1 & Ch1.0 & -0.04 & -3.77 & 0.08 & 0.47 & -0.00 & 4.67 & 0.09 & 0.55 & -0.03 & -3.23 & 0.08 & 0.48 & -0.00 & 5.55 & 0.09 & 0.56 & -0.83 & 351 \\
\hline SGP-C1 & Ch7.0 & -0.04 & -5.54 & 0.07 & 0.66 & 0.02 & 7.00 & 0.06 & 0.81 & -0.03 & -4.89 & 0.07 & 0.71 & 0.02 & 6.23 & 0.06 & 0.88 & -1.53 & 128 \\
\hline SGP-C1 & Ch4.5 & -0.02 & -1.64 & 0.08 & 0.66 & 0.03 & 12.81 & 0.08 & 0.83 & -0.02 & -2.11 & 0.08 & 0.69 & 0.03 & 12.63 & 0.08 & 0.87 & -1.49 & 270 \\
\hline SGP-C1 & Ch3.0 & -0.02 & -0.26 & 0.09 & 0.63 & 0.03 & 17.46 & 0.09 & 0.80 & -0.02 & -0.63 & 0.09 & 0.65 & 0.03 & 17.60 & 0.09 & 0.83 & -1.77 & 451 \\
\hline SGP-C1 & Ch1.8 & -0.03 & -0.24 & 0.09 & 0.62 & 0.01 & 14.01 & 0.09 & 0.73 & -0.03 & -0.39 & 0.09 & 0.64 & 0.01 & 14.16 & 0.09 & 0.75 & -1.86 & 493 \\
\hline SGP-C1 & Ch1.0 & -0.03 & -1.77 & 0.09 & 0.58 & -0.01 & 10.20 & 0.09 & 0.64 & -0.03 & -1.89 & 0.09 & 0.59 & -0.01 & 10.39 & 0.09 & 0.65 & -1.70 & 533 \\
\hline TWP-C1 & Ch7.0 & -0.01 & -0.69 & 0.06 & 0.83 & 0.02 & 3.99 & 0.08 & 0.90 & 0.00 & 0.84 & 0.06 & 0.91 & 0.04 & 6.38 & 0.09 & 0.99 & -1.83 & 312 \\
\hline TWP-C1 & Ch4.5 & -0.04 & -5.07 & 0.06 & 0.72 & -0.04 & -4.70 & 0.08 & 0.80 & -0.03 & -4.16 & 0.06 & 0.77 & -0.03 & -3.14 & 0.09 & 0.86 & -1.24 & 312 \\
\hline TWP-C1 & Ch3.0 & -0.06 & -8.68 & 0.07 & 0.65 & -0.09 & -12.10 & 0.10 & 0.67 & -0.06 & -8.18 & 0.07 & 0.68 & -0.08 & -11.26 & 0.10 & 0.70 & -1.10 & 312 \\
\hline TWP-C1 & Ch1.8 & -0.06 & -9.88 & 0.07 & 0.64 & -0.11 & -15.25 & 0.11 & 0.58 & -0.06 & -9.52 & 0.07 & 0.66 & -0.10 & -14.70 & 0.11 & 0.60 & -0.88 & 312 \\
\hline TWP-C1 & Ch1.0 & -0.05 & -7.89 & 0.07 & 0.63 & -0.10 & -13.26 & 0.13 & 0.52 & -0.05 & -7.58 & 0.07 & 0.64 & -0.10 & -12.78 & 0.12 & 0.53 & -0.30 & 312 \\
\hline TWP-C2 & Ch7.0 & 0.00 & 1.41 & 0.06 & 0.77 & 0.02 & 5.61 & 0.07 & 0.83 & 0.00 & 1.60 & 0.06 & 0.83 & 0.03 & 6.15 & 0.07 & 0.89 & -1.74 & 430 \\
\hline TWP-C2 & Ch4.5 & -0.02 & -1.06 & 0.06 & 0.69 & -0.02 & -1.89 & 0.07 & 0.75 & -0.02 & -1.04 & 0.06 & 0.73 & -0.02 & -1.41 & 0.07 & 0.79 & -1.32 & 436 \\
\hline TWP-C2 & Ch3.0 & -0.03 & -4.31 & 0.06 & 0.65 & -0.05 & -7.49 & 0.08 & 0.66 & -0.03 & -4.49 & 0.06 & 0.68 & -0.05 & -7.32 & 0.08 & 0.68 & -1.37 & 436 \\
\hline TWP-C2 & Ch1.8 & -0.04 & -6.37 & 0.06 & 0.64 & -0.05 & -7.31 & 0.10 & 0.58 & -0.04 & -6.47 & 0.06 & 0.66 & -0.05 & -7.17 & 0.09 & 0.59 & -1.44 & 436 \\
\hline TWP-C2 & Ch1.0 & -0.04 & -5.41 & 0.06 & 0.64 & -0.04 & -0.62 & 0.11 & 0.52 & -0.04 & -5.40 & 0.06 & 0.65 & -0.04 & -0.35 & 0.10 & 0.53 & -1.10 & 436 \\
\hline TWP-C3 & Ch7.0 & 0.23 & 55.96 & 0.13 & 0.65 & 0.47 & 104.99 & 0.17 & 0.77 & 0.40 & 93.72 & 0.17 & 0.68 & 0.82 & 183.78 & 0.24 & 0.83 & -3.34 & 28 \\
\hline TWP-C3 & Ch4.5 & 0.15 & 42.00 & 0.11 & 0.61 & 0.42 & 108.10 & 0.16 & 0.67 & 0.23 & 60.68 & 0.13 & 0.65 & 0.64 & 163.11 & 0.20 & 0.72 & -2.91 & 35 \\
\hline TWP-C3 & Ch3.0 & 0.09 & 30.69 & 0.11 & 0.56 & 0.29 & 87.71 & 0.15 & 0.55 & 0.13 & 40.23 & 0.11 & 0.59 & 0.41 & 117.93 & 0.17 & 0.57 & -3.45 & 39 \\
\hline TWP-C3 & Ch1.8 & 0.05 & 21.74 & 0.10 & 0.54 & 0.13 & 51.36 & 0.15 & 0.45 & 0.07 & 25.73 & 0.10 & 0.56 & 0.17 & 61.76 & 0.15 & 0.46 & -3.91 & 39 \\
\hline TWP-C3 & Ch1.0 & 0.02 & 13.10 & 0.08 & 0.59 & 0.03 & 25.96 & 0.14 & 0.42 & 0.03 & 14.34 & 0.08 & 0.60 & 0.05 & 28.65 & 0.14 & 0.43 & -3.82 & 39 \\
\hline
\end{tabular}

